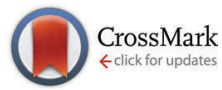

Cite this: Phys. Chem. Chem. Phys., 2016, 18, 20955

Received 18th January 2016, Accepted 3rd March 2016 DOI: $10.1039 / c 6 c p 00392 c$

www.rsc.org/pccp

\title{
Analytical gradients for excitation energies from frozen-density embedding $\dagger$
}

\author{
Arseny Kovyrshin ${ }^{\mathrm{a}}$ and Johannes Neugebauer* ${ }^{\mathrm{b}}$
}

\begin{abstract}
The formulation of analytical excitation-energy gradients from time-dependent density functional theory within the frozen-density embedding framework is presented. In addition to a comprehensive mathematical derivation, we discuss details of the numerical implementation in the Slater-function based Amsterdam Density Functional (ADF) program. Particular emphasis is put on the consistency in the use of approximations for the evaluation of second-and third-order non-additive kinetic-energy and exchange-correlation functional derivatives appearing in the final expression for the excitationenergy gradient. We test the implementation for different chemical systems in which molecular excitedstate potential-energy curves are affected by another subsystem. It is demonstrated that the analytical implementation for the evaluation of excitation-energy gradients yields results in close agreement with data from numerical differentiation. In addition, we show that our analytical results are numerically more stable and thus preferable over the numerical ones.
\end{abstract}

\section{Introduction}

A full interpretation of excited-state processes requires to have reliable theoretical models for the explanation of experimental data in terms of excited-state geometric and electronic structure at hand. ${ }^{1}$ The characterization of excited-state potential energy surfaces is important for understanding a variety of phenomena, such as photochemical reactions, vibrationally resolved UV-Vis spectra, resonance Raman spectra, excited-state dynamics etc. Efficient calculations of potential energy surfaces require an analytical approach for the evaluation of excited-state gradients. The main advantage of such an analytical calculation of gradients in contrast to a numerical approach is that the computational cost does not depend on the number of nuclear degrees of freedom. For a long time, the standard method to calculate nuclear derivatives of excited-state energies for large molecular systems has been the configuration interaction singles method. ${ }^{2}$ In 1994, Ortiz derived and implemented gradients of excited states within the random phase approximation. ${ }^{3}$ The first implementation of analytical excited-state gradients within TimeDependent Density Functional Theory (TDDFT) was presented by Van Caillie and Amos. ${ }^{4}$ While in that work the expression for the gradients was obtained by straightforward differentiation,

\footnotetext{
${ }^{a}$ Laboratorium für Physikalische Chemie, ETH Zürich, Vladimir-Prelog-Weg 2, 8093 Zürich, Switzerland. E-mail: j.neugebauer@uni-muenster.de

${ }^{b}$ Theoretische Organische Chemie, Organisch-Chemisches Institut and Center for Multiscale Theory and Simulation, Westfälische Wilhelms-Universität Münster, Corrensstraße 40, 48149 Münster, Germany

$\dagger$ Dedicated to Professor Evert Jan Baerends on the occasion of his 70th birthday.
}

Furche and Ahlrichs employed ${ }^{5}$ a more convenient Lagrangianbased approach, similar to the one used in ref. 6 for configuration interaction methods. The same technique was used by Hutter in his work, ${ }^{7}$ where the calculation of analytical excited-state gradients from the Tamm-Dancoff approximation to TDDFT was implemented within a plane-wave-basis set framework. In 2005, Doltsinis extended ${ }^{8}$ plane-wave TDDFT excited-state gradient calculations beyond the Tamm-Dancoff approximation, where in addition, an active space approach ${ }^{9}$ and an implicit differentiation scheme were employed. Seth et al. ${ }^{10}$ implemented analytical gradients for spin-flip TDDFT excitation in the Amsterdam Density Functional program package (ADF), ${ }^{11,12}$ which uses Slatertype functions in contrast to most other quantum chemistry packages. These implementations provided a powerful toolbox for the theoretical characterization of excited-state potentialenergy surfaces of large molecules. Embedding techniques like the Polarizable Continuum Model $^{13}$ (PCM), Quantum Mechanics/Molecular Mechanics ${ }^{14}$ (QM/MM) or Frozen-Density Embedding $(\mathrm{FDE})^{15}$ can increase the range of application for TDDFT gradients. Analytical gradients for the evaluation of excitation-energy gradients have been implemented, e.g. for PCM-TDDFT $^{16}$ and QM/MM-TDDFT ${ }^{17}$ and other fragmentation or embedding strategies. ${ }^{18,19}$ However, no such analytical gradients are available so far for FDE, which partitions the total electron density of a large system into that of an active region and the frozen density of an environment. The TDDFT extension of FDE has been presented in ref. 20 and 21, and several challenging applications have been presented since then (see ref. 22-25 or the review in ref. 26 for some examples). Following the strict version of "frozen-density embedding", we will assume here and in 
the following that the response of the system to an external perturbation is entirely localized in the active system. This corresponds to the case of "uncoupled FDE" (FDEu) for excited states in the nomenclature of ref. 27 , which is also known as the "neglect of differential response of the environment" (NDRE). ${ }^{28}$ This can be relaxed in a full subsystem TDDFT treatment (see ref. 27 and the work by Casida and Wesolowski ${ }^{20}$ ). For recent reviews, see ref. 29 and 30. Note that analytical ground-state energy gradients for FDE have been reported before, ${ }^{31}$ and will thus not be considered here. The development of analytical excited-state gradient theory in TDDFT within FDE will give fast access to potential energy surfaces of the excited states of complex systems.

\section{Theory}

The excited-state gradient in TDDFT is calculated as the sum of the ground-state gradient and the gradient of the excitation energy, but since the former is established for $\mathrm{FDE}^{31}$ we focus here only on the latter part. In order to introduce the notation, the underlying theory of FDE and its TDDFT extension are briefly presented in Sections 2.1 and 2.2, respectively. The formulation of FDEu-TDDFT excitation-energy gradients is concisely established in Section 2.3, which is followed by a derivation of the $Z$-vector equation for FDEu-TDDFT gradients in Section 2.4. An in-depth derivation including all intermediate steps is presented in Appendix A. Note that our theoretical development is based on the formalism introduced in the work by Seth et al. ${ }^{10}$ as well as in that by Furche and Ahlrichs. ${ }^{5}$

\subsection{Frozen-density embedding}

The FDE theory ${ }^{15}$ is a fundamental framework for embedding schemes within DFT. The total density of the system in the FDE approach is partitioned into an active part and a frozen part

$$
\rho_{\text {tot }}(\mathbf{r})=\rho(\mathbf{r})+\rho_{\mathrm{f}}(\mathbf{r}) .
$$

The active electron density, $\rho(\mathbf{r})$, can be expressed through the orbitals located in the active fragment of the molecular system,

$$
\rho(\mathbf{r})=\sum_{\mathrm{i}}^{N} \phi_{\mathrm{i}}^{*}(\mathbf{r}) \phi_{\mathrm{i}}(\mathbf{r}),
$$

where $N$ is the number of electrons in the active part. The molecular orbitals of this fragment are usually varied during the calculation, and hence its density as well. Contrarily, the density of the frozen part, $\rho_{\mathrm{f}}(\mathbf{r})$, is chosen to be constraint during the calculation. In the context of the related subsystem DFT method, ${ }^{32-34}$ it consists of orbitals located in the environment, $\phi_{\mathrm{i}_{\mathrm{f}}}$,

$$
\rho_{\mathrm{f}}(\mathbf{r})=\sum_{\mathrm{i}_{\mathrm{f}}}^{N_{\mathrm{f}}} \phi_{\mathrm{i}_{\mathrm{f}}}^{*}(\mathbf{r}) \phi_{\mathrm{i}_{\mathrm{f}}}(\mathbf{r}),
$$

and both subsystem densities can be optimized. Please note that we do not use an extra label for indices of orbitals, densities, and potentials of the active fragment, while the terms for the frozen fragment are marked with "f" as a subscript (or, in the case of potentials, as a superscript).
In order to obtain the ground-state density of the active system, $\rho(\mathbf{r})$, in the presence of the frozen density, the Kohn-Sham (KS) equations with Constrained Electron Density (KSCED) ${ }^{15}$ must be solved,

$$
\left(-\frac{1}{2} \nabla^{2}+v^{\mathrm{eff}}[\rho](\mathbf{r})+v^{\mathrm{emb}}\left[\rho, \rho_{\mathrm{f}}\right](\mathbf{r})\right) \phi_{\mathrm{i}}=\varepsilon_{\mathrm{i}} \phi_{\mathrm{i}},
$$

where $v^{\text {eff }}[\rho](\mathbf{r})$ has the same form as the effective potential for the standard Kohn-Sham equation of the isolated active system,

$$
v^{\mathrm{eff}}[\rho](\mathbf{r})=\underbrace{-\sum_{\mathrm{A}} \frac{Z_{\mathrm{A}}}{\left|\mathbf{r}-\mathbf{R}_{\mathrm{A}}\right|}}_{v_{\mathrm{ext}}}+\underbrace{\int \frac{\rho\left(\mathbf{r}^{\prime}\right)}{\left|\mathbf{r}^{\prime}-\mathbf{r}\right|} \mathrm{d} \mathbf{r}^{\prime}}_{v_{\mathrm{C}}}+\underbrace{\frac{\delta E_{\mathrm{XC}}[\rho]}{\delta \rho(\mathbf{r})}}_{v_{\mathrm{XC}}},
$$

and $v^{\mathrm{emb}}\left[\rho, \rho_{\mathrm{f}}\right](\mathbf{r})$ is responsible for the interaction of the active part with the frozen environment density,

$$
\begin{aligned}
v^{\mathrm{emb}}\left[\rho, \rho_{\mathrm{f}}\right](\mathbf{r})= & -\underbrace{-\sum_{A_{\mathrm{f}}} \frac{Z_{A_{\mathrm{f}}}}{\left|\mathbf{r}-\mathbf{R}_{A_{\mathrm{f}}}\right|}}_{v_{\mathrm{ext}}^{\mathrm{f}}}+\underbrace{\int \frac{\rho_{\mathrm{f}}\left(\mathbf{r}^{\prime}\right)}{\left|\mathbf{r}^{\prime}-\mathbf{r}\right|} \mathrm{d} \mathbf{r}^{\prime}}_{v_{\mathrm{C}}^{\mathrm{f}}}+v_{\mathrm{XC}}^{\mathrm{nadd}}\left[\rho, \rho_{\mathrm{f}}\right](\mathbf{r}) \\
& +v_{\text {kin }}^{\text {nadd }}\left[\rho, \rho_{\mathrm{f}}\right](\mathbf{r}) .
\end{aligned}
$$

The last two terms represent the so-called non-additive XC potential and non-additive kinetic potential, which read

$$
\begin{gathered}
v_{\mathrm{XC}}^{\text {nadd }}\left[\rho, \rho_{\mathrm{f}}\right](\mathbf{r})=\frac{\delta E_{\mathrm{XC}}\left[\rho_{\mathrm{tot}}\right]}{\delta \rho_{\mathrm{tot}}(\mathbf{r})}-\frac{\delta E_{\mathrm{XC}}[\rho]}{\delta \rho(\mathbf{r})}, \\
v_{\text {kin }}^{\text {nadd }}\left[\rho, \rho_{\mathrm{f}}\right](\mathbf{r})=\frac{\delta T_{\mathrm{s}}\left[\rho_{\mathrm{tot}}\right]}{\delta \rho_{\mathrm{tot}}(\mathbf{r})}-\frac{\delta T_{\mathrm{s}}[\rho]}{\delta \rho(\mathbf{r})} .
\end{gathered}
$$

The term in parenthesis on the left hand-side of eqn (4) is denoted as $\hat{F}$ and can be identified as the time-independent FDE-KS Hamiltonian.

\subsection{Frozen-density embedding within TDDFT}

The FDE formalism has been extended to excited states by Casida and Wesolowski, ${ }^{20}$ and a general subsystem TDDFT framework was established in ref. 27. The FDE approach can be considered as a special case of the subsystem-based TDDFT scheme in which local excitations are obtained under the influence of a given environment, e.g. a solvent. The matrix elements of the timeindependent FDE-KS Hamiltonian are given as

$$
F_{\mathrm{pq}}=\int \phi_{\mathrm{p}}{ }^{*}(\mathbf{r})\left[-\frac{1}{2} \nabla^{2}+v^{\mathrm{eff}}[\rho](\mathbf{r})+v^{\mathrm{emb}}\left[\rho, \rho_{\mathrm{f}}\right](\mathbf{r})\right] \phi_{\mathrm{q}}(\mathbf{r}) \mathrm{d} \mathbf{r},
$$

where $\phi_{\mathrm{p}}$ are orbitals of the active part of the system. The convention that indices $\mathrm{i}, \mathrm{j}$, etc. correspond to occupied orbitals, $\mathrm{a}, \mathrm{b}$, etc. to virtual orbitals and p,q, etc. refer to general orbitals is adopted for the following derivations. Expanding the timedependent one-electron functions, $\phi_{\mathrm{p}}(\mathbf{r}, t)$, in a linear combination of time-independent one-electron functions, $\phi_{\mathrm{j}}(\mathbf{r})$ with time-dependent orbital coefficients, $K_{\mathrm{jp}}(t)$,

$$
\phi_{\mathrm{p}}(\mathbf{r}, t)=\sum_{\mathrm{j}}^{M} K_{\mathrm{jp}}(t) \phi_{\mathrm{j}}(\mathbf{r}),
$$


the Time-Dependent KS (TDKS) equations will take the following form ${ }^{35,36}$

$$
i \frac{\partial}{\partial t} \mathbf{K}=\mathbf{F K}
$$

where the $i$-th column of the matrix $\mathbf{K}$ contains the expansion coefficients of $\phi_{\mathrm{i}}(\mathbf{r}, t)$. F is a matrix representation of the TDKS Hamiltonian operator in the basis of $\left\{\phi_{\mathrm{i}}(\mathbf{r})\right\}$. Assuming that a time-dependent external field is applied ${ }^{35}$ one can derive the main equation of linear-response TDDFT within the FDE approach from eqn (11),

$$
\left[\begin{array}{ll}
\mathbf{A} & \mathbf{B} \\
\mathbf{B} & \mathbf{A}
\end{array}\right]\left[\begin{array}{l}
\mathbf{X} \\
\mathbf{Y}
\end{array}\right]=\omega\left[\begin{array}{cc}
\mathbf{1} & \mathbf{0} \\
\mathbf{0} & -\mathbf{1}
\end{array}\right]\left[\begin{array}{l}
\mathbf{X} \\
\mathbf{Y}
\end{array}\right]
$$

where the elements of $\mathbf{A}$ and $\mathbf{B}$ read (real orbitals are assumed),

$$
\begin{aligned}
A_{\mathrm{ia}, \mathrm{jb}}= & F_{\mathrm{ab}} \delta_{\mathrm{ij}}-F_{\mathrm{ij}} \delta_{\mathrm{ab}}+(\mathrm{ia} \mid \mathrm{jb})+\left(\mathrm{ia}\left|f_{\mathrm{XC}}\right| \mathrm{jb}\right)+\left(\mathrm{ia}\left|f_{\mathrm{kin}}^{\text {nadd }}\right| \mathrm{jb}\right) \\
& +\left(\mathrm{ia}\left|f_{\mathrm{XC}}^{\text {nadd }}\right| \mathrm{jb}\right), \\
B_{\mathrm{ia}, \mathrm{jb}}= & (\mathrm{ia} \mid \mathrm{bj})+\left(\mathrm{ia}\left|f_{\mathrm{XC}}\right| \mathrm{bj}\right)+\left(\mathrm{ia}\left|f_{\mathrm{kin}}^{\text {nadd }}\right| \mathrm{bj}\right)+\left(\mathrm{ia}\left|f_{\mathrm{XC}}^{\text {nadd }}\right| \mathrm{bj}\right) .
\end{aligned}
$$

The response of the XC potential corresponds to the second functional derivative of the exchange-correlation energy, which is called XC kernel and given as

$$
\left(\mathrm{ia}\left|f_{\mathrm{XC}}\right| \mathrm{jb}\right)=\iint \phi_{\mathrm{i}}(\mathbf{r}) \phi_{\mathrm{a}}(\mathbf{r}) \frac{\delta^{2} E^{\mathrm{XC}}[\rho]}{\delta \rho(\mathbf{r}) \delta \rho\left(\mathbf{r}^{\prime}\right)} \phi_{\mathrm{b}}\left(\mathbf{r}^{\prime}\right) \phi_{\mathrm{j}}\left(\mathbf{r}^{\prime}\right) \mathrm{d} \mathbf{r} \mathrm{d} \mathbf{r}^{\prime} .
$$

Only non-additive terms from the embedding potential, $v^{\mathrm{emb}}\left[\rho, \rho_{\mathrm{f}}\right](\mathbf{r})$, remain and they are represented as a second functional derivative of the corresponding non-additive energies, which are also called non-additive kernels,

$$
\begin{aligned}
\left(\mathrm{i} a\left|f_{\mathrm{XC}}^{\mathrm{nadd}}\right| \mathrm{jb}\right)= & \int \phi_{\mathrm{i}}^{*}(\mathbf{r}) \phi_{\mathrm{a}}(\mathbf{r})\left(\frac{\delta^{2} E_{\mathrm{XC}}\left[\rho_{\mathrm{tot}}\right]}{\delta \rho_{\mathrm{tot}}(\mathbf{r}) \delta \rho_{\mathrm{tot}}\left(\mathbf{r}^{\prime}\right)}-\frac{\delta^{2} E_{\mathrm{XC}}[\rho]}{\delta \rho(\mathbf{r}) \delta \rho\left(\mathbf{r}^{\prime}\right)}\right) \\
& \times \phi_{\mathrm{b}}{ }^{*}\left(\mathbf{r}^{\prime}\right) \phi_{\mathrm{j}}\left(\mathbf{r}^{\prime}\right) \mathrm{d} \mathbf{r} \mathrm{d} \mathbf{r}^{\prime},
\end{aligned}
$$

$$
\begin{aligned}
\left(\mathrm{ia}\left|f_{\text {kin }}^{\text {nadd }}\right| \mathrm{jb}\right)= & \int \phi_{\mathrm{i}}^{*}(\mathbf{r}) \phi_{\mathrm{a}}(\mathbf{r})\left(\frac{\delta^{2} T_{\mathrm{s}}\left[\rho_{\text {tot }}\right]}{\delta \rho_{\text {tot }}(\mathbf{r}) \delta \rho_{\text {tot }}\left(\mathbf{r}^{\prime}\right)}-\frac{\delta^{2} T_{\mathrm{s}}[\rho]}{\delta \rho(\mathbf{r}) \delta \rho\left(\mathbf{r}^{\prime}\right)}\right) \\
& \times \phi_{\mathrm{b}}{ }^{*}\left(\mathbf{r}^{\prime}\right) \phi_{\mathrm{j}}\left(\mathbf{r}^{\prime}\right) \mathrm{d} \mathbf{r} \mathrm{d} \mathbf{r}^{\prime} .
\end{aligned}
$$

Using the fact that in the basis of canonical orbitals

$$
\begin{gathered}
F_{\mathrm{ab}}=F_{\mathrm{ab}} \delta_{\mathrm{ab}}=\varepsilon_{\mathrm{a}} \delta_{\mathrm{ab}}, \\
F_{\mathrm{ij}}=F_{\mathrm{ij}} \delta_{\mathrm{ij}}=\varepsilon_{\mathrm{i}} \delta_{\mathrm{ij}},
\end{gathered}
$$

elements of matrices $\mathbf{A}$ and $\mathbf{B}$ change to

$$
\begin{aligned}
A_{\mathrm{ia}, \mathrm{jb}}= & \delta_{\mathrm{ij}} \delta_{\mathrm{ab}}\left(\varepsilon_{\mathrm{a}}-\varepsilon_{\mathrm{i}}\right)+(\mathrm{ia} \mid \mathrm{jb})+\left(\mathrm{ia}\left|f_{\mathrm{XC}}\right| \mathrm{jb}\right)+\left(\mathrm{ia}\left|f_{\mathrm{kin}}^{\text {nadd }}\right| \mathrm{jb}\right) \\
& +\left(\mathrm{ia}\left|f_{\mathrm{XC}}^{\text {nadd }}\right| \mathrm{jb}\right), \\
B_{\mathrm{ia}, \mathrm{jb}}= & (\mathrm{ia} \mid \mathrm{bj})+\left(\mathrm{ia}\left|f_{\mathrm{XC}}\right| \mathrm{bj}\right)+\left(\mathrm{ia}\left|f_{\mathrm{kin}}^{\text {nadd }}\right| \mathrm{bj}\right)+\left(\mathrm{ia}\left|f_{\mathrm{XC}}^{\text {nadd }}\right| \mathrm{bj}\right) .
\end{aligned}
$$

In the ADF package $\mathrm{XC}$, non-additive $\mathrm{XC}$, and non-additive kinetic kernels in the equations above are by default evaluated within the adiabatic local density approximation (ALDA) for non-hybrid XC potentials. This gives a good compromise between accuracy and efficiency. Moreover, ALDA kernels show smoother behavior for the low density regions than adiabatic generalized gradient approximation (AGGA) kernels. The last statement can be explained by the absence of $1 /|\nabla \rho(\mathbf{r})|^{\beta}$ dependencies in kernels, where $\beta$ is a positive exponent.

We note that it is sometimes useful to rearrange eqn (12) by means of the unitary transformation $\mathbf{U}$,

$$
\mathbf{U}=\frac{1}{\sqrt{2}}\left(\begin{array}{cc}
1 & 1 \\
-1 & 1
\end{array}\right)
$$

into the two coupled equations

$$
\begin{aligned}
& (\mathbf{A}+\mathbf{B})(\mathbf{X}+\mathbf{Y})=\omega(\mathbf{X}-\mathbf{Y}), \\
& (\mathbf{A}-\mathbf{B})(\mathbf{X}-\mathbf{Y})=\omega(\mathbf{X}+\mathbf{Y}) .
\end{aligned}
$$

\subsection{TDDFT excitation-energy gradients within FDE}

Having the concepts of excitation-energy gradients for regular TDDFT at hand, ${ }^{5,10}$ it is possible to establish the theory of FDEuTDDFT excited-state gradients in a similar way (see Appendix A for a detailed derivation).

The Lagrangian for FDEu-TDDFT reads [see eqn (86) in Appendix A.2]

$$
\begin{aligned}
L[\mathbf{X}, \mathbf{Y}, \omega, \mathbf{C}, \mathbf{Z}, \mathbf{W}]= & G[\mathbf{X}, \mathbf{Y}, \omega]+\sum_{\mathrm{ia}} Z_{\mathrm{ia}} F_{\mathrm{ia}} \\
& -\sum_{\mathrm{pq}, \mathrm{p} \leq \mathrm{q}} W_{\mathrm{pq}}\left(S_{\mathrm{pq}}-\delta_{\mathrm{pq}}\right) .
\end{aligned}
$$

The quantity $G[\mathbf{X}, \mathbf{Y}, \omega]$ has the following form,

$$
G[\mathbf{X}, \mathbf{Y}, \omega]=\left[\mathbf{X}^{\dagger} \mathbf{Y}^{\dagger}\right]\left[\begin{array}{ll}
\mathbf{A} & \mathbf{B} \\
\mathbf{B} & \mathbf{A}
\end{array}\right]\left[\begin{array}{l}
\mathbf{X} \\
\mathbf{Y}
\end{array}\right]-\omega\left(\left[\mathbf{X}^{\dagger} \mathbf{Y}^{\dagger}\right]\left[\begin{array}{c}
\mathbf{X} \\
-\mathbf{Y}
\end{array}\right]-1\right),
$$

where $\mathbf{A}$ and $\mathbf{B}$ are the matrices specified in Section 2.2, $\mathbf{X}$ and $\mathbf{Y}$ are the solution vectors from eqn (12), $\mathbf{C}$ is the matrix of orbital coefficients. $\mathbf{Z}$ and $\mathbf{W}$ in eqn (25) contain two additional sets of Lagrangian multipliers. The sum $\sum_{\mathrm{ia}} Z_{\mathrm{ia}} F_{\mathrm{ia}}$ takes care of the conditions $F_{\text {ia }}=0$ (which implies that KSCED equations are solved, but not necessarily in canonical orbitals) and $\sum_{\mathrm{pq}, \mathrm{p} \leq \mathrm{q}} W_{\mathrm{pq}}\left(S_{\mathrm{pq}}-\delta_{\mathrm{pq}}\right)$ ensures orthonormality of the orbitals ( $S_{\mathrm{pq}}$ is the overlap integral for orbitals $\mathrm{p}$ and $\mathrm{q}$ ). Note that our definition of the vector $\mathbf{Z}$ implies that only occupied-virtual components among all $Z_{\mathrm{rs}}$ are non-zero. The FDE-TDDFT eigenvalue problem can be expressed as

$$
\frac{\partial L[\mathbf{X}, \mathbf{Y}, \omega, \mathbf{C}, \mathbf{Z}, \mathbf{W}]}{\partial\left[\mathbf{X}^{\dagger} \mathbf{Y}^{\dagger}\right]}=\frac{\partial G[\mathbf{X}, \mathbf{Y}, \omega]}{\partial\left[\mathbf{X}^{\dagger} \mathbf{Y}^{\dagger}\right]}=0,
$$

and the orthonormality constraint is given as

$$
\frac{\partial L[\mathbf{X}, \mathbf{Y}, \omega, \mathbf{C}, \mathbf{Z}, \mathbf{W}]}{\partial \omega}=\frac{\partial G[\mathbf{X}, \mathbf{Y}, \omega]}{\partial \omega}=0 .
$$


It is possible to rewrite the Lagrangian as (see Appendix A.3; for simplicity of notation the Lagrangian, $L[\mathbf{X}, \mathbf{Y}, \omega, \mathbf{C}, \mathbf{Z}, \mathbf{W}]$, will be written from now on as $L$ )

$$
\begin{aligned}
L= & \sum_{\mathrm{pq}} F_{\mathrm{pq}} P_{\mathrm{pq}}-\sum_{\mathrm{pq}, \mathrm{p} \leq \mathrm{q}}\left(S_{\mathrm{pq}}-\delta_{\mathrm{pq}}\right) W_{\mathrm{pq}} \\
& +\sum_{\mathrm{ia}, \mathrm{jb}}(X+Y)_{\mathrm{ia}}(X+Y)_{\mathrm{jb}}[(\mathrm{ia} \mid \mathrm{jb}) \\
& \left.+f_{\mathrm{ia}, \mathrm{jb}}^{\mathrm{XC}}+f_{\mathrm{XC}, \mathrm{ia}, \mathrm{jb}}^{\mathrm{nadd}}+f_{\mathrm{kin}, \mathrm{ia}, \mathrm{jb}}^{\text {nadd }}\right] \\
& -\omega\left[\sum_{\mathrm{ia}}(X+Y)_{\mathrm{ia}}(X-Y)_{\mathrm{ia}}-1\right] .
\end{aligned}
$$

In the equation above, a relaxed difference density matrix $\mathbf{P}$ was introduced (see Appendix A.6 for more details),

$$
\mathbf{P}=\mathbf{T}+\mathbf{Z},
$$

where $\mathbf{T}$ is an unrelaxed difference density matrix with elements equal to

$$
\begin{aligned}
& T_{\mathrm{ab}}=\frac{1}{2} \sum_{\mathrm{i}}\left\{(X+Y)_{\mathrm{ia}}(X+Y)_{\mathrm{ib}}+(X-Y)_{\mathrm{ia}}(X-Y)_{\mathrm{ib}}\right\}, \\
& T_{\mathrm{ij}}=-\frac{1}{2} \sum_{\mathrm{a}}\left\{(X+Y)_{\mathrm{ia}}(X+Y)_{\mathrm{ja}}+(X-Y)_{\mathrm{ia}}(X-Y)_{\mathrm{ja}}\right\},
\end{aligned}
$$

$$
T_{\mathrm{ia}}=T_{\mathrm{ai}}=0 .
$$

We now introduce some external perturbation $\xi$. In our particular case, $\xi$ represents some nuclear coordinate $R_{\mathrm{A}_{k}}$ (i.e., component $k$ of the position vector of atom A). The derivatives of the Lagrangian with respect to all possible nuclear coordinates $\xi$ in the system constitute the gradient of the excitation energy with respect to the position of the nuclei. As stressed by Furche and Ahlrichs, the formalism is also valid if $\xi$ represents some general perturbation, e.g. a component of a static electric field, which gives access to the excited-state dipole moment. ${ }^{5}$ In order to differentiate the Lagrangian with respect to a perturbation $\xi$, it is useful first to convert it to atomic orbital representation (see also Appendices A.4 and A.5). In comparison with the regular TDDFT case, eqn (29) contains the additional terms $\sum_{\mathrm{ia}, \mathrm{jb}}(X+Y)_{\mathrm{ia}}(X+Y)_{\mathrm{jb}}\left[f_{\mathrm{XC}, \mathrm{ia}, \mathrm{jb}}^{\text {nadd }}+f_{\mathrm{kin}, \mathrm{ia}, \mathrm{jb}}^{\text {nadd }}\right]$ (which results from $G[\mathbf{X}, \mathbf{Y}, \omega])$ and $\sum_{\mathrm{pq}} v_{\mathrm{pq}}^{\mathrm{emb}}\left[\rho, \rho_{\mathrm{f}}\right](\mathbf{r}) P_{\mathrm{pq}} \quad$ (which results from $\left.\sum_{\mathrm{pq}} F_{\mathrm{pq}} P_{\mathrm{pq}}\right)$. The focus here will thus be mostly on these terms. For convenience, we will sometimes drop the notation $v^{\text {emb }}\left[\rho, \rho_{\mathrm{f}}\right], v^{\text {eff }}[\rho]$, etc. and write $v^{\text {emb }}, v^{\text {eff }}$ instead. Converting $\sum_{\mathrm{pq}} F_{\mathrm{pq}} P_{\mathrm{pq}}$ to atomic orbital basis, we get

$$
\sum_{\mathrm{pq}} F_{\mathrm{pq}} P_{\mathrm{pq}}=\sum_{\mu v} F_{\mu v} \sum_{\mathrm{pq}} C_{\mu \mathrm{p}} C_{\mathrm{vq}} P_{\mathrm{pq}}=\sum_{\mu \nu} F_{\mu \nu} P_{\mu v} .
$$

For convenience, we define the kinetic energy operator $\hat{T}_{\mathrm{s}}=-\frac{1}{2} \nabla^{2}$. Keeping the orbital coefficients constant and taking the derivative with respect to the perturbation $\xi[\xi$ as a superscript indicates differentiation over $\xi$, while $(\xi)$ means that MO coefficients are kept constant during differentiation], we get from the equation above,

$$
\begin{aligned}
\left(\sum_{\mu \nu} F_{\mu v} P_{\mu \nu}\right)^{(\xi)}= & \sum_{\mu \nu}\left(\chi_{\mu} \hat{T}_{\mathrm{s}} \chi_{v}\right)^{\xi} P_{\mu \nu}+\sum_{\mu \nu}\left(v_{\mu \nu}^{\mathrm{eff}}\right)^{(\xi)} P_{\mu \nu} \\
& +\sum_{\mu \nu}\left(v_{\mu \nu}^{\mathrm{emb}}\right)^{(\xi)} P_{\mu \nu}
\end{aligned}
$$

The first term reads ${ }^{10}$

$$
\int \sum_{\mu \nu}\left(\chi_{\mu} \hat{T}_{\mathrm{s}} \chi_{v}\right)^{\xi} P_{\mu \nu}=T_{\mathrm{s}}\left[\rho^{\mathrm{P}(\xi)}\right] .
$$

Here, we introduced the density

$$
\rho^{\mathrm{P}}=\sum_{\mu \nu} P_{\mu v} \chi_{\mu} \chi_{v}
$$

The second term $\sum_{\mu v}\left(v_{\mu v}^{\text {eff }}\right)^{(\xi)}$ reads (here, we introduce a simplified notation in which the integration of a function $\int f(\mathbf{r}) \mathrm{d} \mathbf{r}$ or $\int f\left(\mathbf{r}, \mathbf{r}^{\prime}\right) \mathrm{d} \mathbf{r} \mathrm{d} \mathbf{r}^{\prime}$ will be expressed as $\left.\int f\right)$,

$$
\begin{aligned}
\sum_{\mu \nu}\left(v_{\mu \nu}^{\text {eff }}\right)^{(\xi)} P_{\mu \nu}= & -\int \sum_{\mathrm{A}} \rho^{\mathrm{P}(\xi)}(\mathbf{r}) \frac{Z_{\mathrm{A}}}{\left|\mathbf{r}-\mathbf{R}_{\mathrm{A}}\right|} \mathrm{d} \mathbf{r} \\
& -\int \sum_{\mathrm{A}} \rho^{\mathrm{P}}(\mathbf{r}) \frac{Z_{\mathrm{A}}\left(r_{k}-R_{\mathrm{A}_{k}}\right)}{\left|\mathbf{r}-\mathbf{R}_{\mathrm{A}}\right|^{3}} \mathrm{~d} \mathbf{r} \\
& +\iint \frac{\rho^{(\xi)}\left(\mathbf{r}^{\prime}\right) \rho^{\mathrm{P}}(\mathbf{r})}{\left|\mathbf{r}^{\prime}-\mathbf{r}\right|} \mathrm{d} \mathbf{r} \mathrm{d} \mathbf{r}^{\prime} \\
& +\iint \frac{\rho\left(\mathbf{r}^{\prime}\right) \rho^{\mathrm{P}(\xi)}(\mathbf{r})}{\left|\mathbf{r}^{\prime}-\mathbf{r}\right|} \mathrm{d} \mathbf{r} \mathrm{d} \mathbf{r}^{\prime} \\
& +\int \rho^{\mathrm{P}(\xi)}(\mathbf{r}) \frac{\delta E_{\mathrm{XC}}[\rho]}{\delta \rho(\mathbf{r})} \mathrm{d} \mathbf{r} \\
& +\iint \rho^{\mathrm{P}}(\mathbf{r}) \frac{\delta^{2} E_{\mathrm{XC}}[\rho]}{\delta \rho(\mathbf{r}) \delta \rho\left(\mathbf{r}^{\prime}\right)} \rho^{(\xi)}\left(\mathbf{r}^{\prime}\right) \mathrm{d} \mathbf{r} \mathrm{d} \mathbf{r}^{\prime} \\
= & \int \rho^{\mathrm{P}(\xi)} v_{\mathrm{ext}}+\int v_{\mathrm{ext}}^{\xi} \rho^{\mathrm{P}}+\int v_{\mathrm{C}}^{\mathrm{P}} \rho^{(\xi)}+\int \rho^{\mathrm{P}(\xi)} v_{\mathrm{C}} \\
& +\int \rho^{\mathrm{P}(\xi)} v^{\mathrm{XC}}+\int \rho^{\mathrm{P}} f^{\mathrm{XC}} \rho^{(\xi)},
\end{aligned}
$$

where $v_{\mathrm{C}}$ and $v_{\mathrm{C}}^{\mathrm{P}}$ are the Coulomb potentials due to $\rho$ and $\rho^{\mathrm{P}}$, respectively, and

$$
D_{\mu v}=\sum_{\mathrm{i}}^{\mathrm{occ}} C_{\mu \mathrm{i}} C_{\mathrm{vi}}
$$

is an element of the ground-state density matrix. Similarly, we get for the third term $\sum_{\mu \nu}\left(v_{\mu \nu}^{\mathrm{emb}}\right)^{(\xi)} P_{\mu \nu}$, 


$$
\begin{aligned}
& \sum_{\mu v}\left(v_{\mu v}^{\mathrm{emb}}\right)^{(\xi)} P_{\mu v}=-\int \sum_{\mathrm{A}_{\mathrm{f}}} \rho^{\mathrm{P}(\xi)}(\mathbf{r}) \frac{Z_{\mathrm{A}_{\mathrm{f}}}}{\left|\mathbf{r}-\mathbf{R}_{\mathrm{A}_{\mathrm{f}}}\right|} \mathrm{d} \mathbf{r} \\
& +\iint \rho^{\mathrm{P}(\xi)}(\mathbf{r}) \frac{\rho_{\mathrm{f}}\left(\mathbf{r}^{\prime}\right)}{\left|\mathbf{r}^{\prime}-\mathbf{r}\right|} \mathrm{d} \mathbf{r} \mathrm{d} \mathbf{r}^{\prime} \\
& +\int \rho^{\mathrm{P}(\xi)}(\mathbf{r})\left(\frac{\delta E_{\mathrm{XC}}\left[\rho_{\text {tot }}\right]}{\delta \rho_{\text {tot }}(\mathbf{r})}-\frac{\delta E_{\mathrm{XC}}[\rho]}{\delta \rho(\mathbf{r})}\right) \mathrm{d} \mathbf{r} \\
& +\int \rho^{\mathrm{P}(\xi)}(\mathbf{r})\left(\frac{\delta T_{\mathrm{s}}\left[\rho_{\text {tot }}\right]}{\delta \rho_{\text {tot }}(\mathbf{r})}-\frac{\delta T_{\mathrm{s}}[\rho]}{\delta \rho(\mathbf{r})}\right) \mathrm{d} \mathbf{r} \\
& +\iint \rho^{\mathrm{P}}(\mathbf{r})\left(\frac{\delta^{2} E_{\mathrm{XC}}\left[\rho_{\mathrm{tot}}\right]}{\delta \rho_{\text {tot }}(\mathbf{r}) \delta \rho_{\mathrm{tot}}\left(\mathbf{r}^{\prime}\right)}-\frac{\delta^{2} E_{\mathrm{XC}}[\rho]}{\delta \rho(\mathbf{r}) \delta \rho\left(\mathbf{r}^{\prime}\right)}\right) \\
& \times \rho^{(\xi)}\left(\mathbf{r}^{\prime}\right) \mathrm{d} \mathbf{r} \mathrm{d} \mathbf{r}^{\prime} \\
& +\iint \rho^{\mathrm{P}}(\mathbf{r})\left(\frac{\delta^{2} T_{\mathrm{s}}\left[\rho_{\text {tot }}\right]}{\delta \rho_{\text {tot }}(\mathbf{r}) \delta \rho_{\text {tot }}\left(\mathbf{r}^{\prime}\right)}-\frac{\delta^{2} T_{\mathrm{s}}[\rho]}{\delta \rho(\mathbf{r}) \delta \rho\left(\mathbf{r}^{\prime}\right)}\right) \\
& \times \rho^{(\xi)}\left(\mathbf{r}^{\prime}\right) \mathrm{d} \mathbf{r} \mathrm{d} \mathbf{r}^{\prime} \\
& =\int \rho^{\mathrm{P}(\xi)} v_{\mathrm{ext}}^{\mathrm{f}}+\int \rho^{\mathrm{P}(\xi)} v_{\mathrm{C}}^{\mathrm{f}} \\
& +\int \rho^{\mathrm{P}(\xi)} v_{\mathrm{XC}}^{\text {nadd }}+\int \rho^{\mathrm{P}(\xi)} v_{\text {kin }}^{\text {nadd }}+\int \rho^{\mathrm{P}} f_{\mathrm{XC}}^{\text {nadd }} \rho^{(\xi)} \\
& +\int \rho^{\mathrm{P}} f_{\mathrm{kin}}^{\mathrm{nadd}} \rho^{(\xi)},
\end{aligned}
$$

where $v_{\mathrm{C}}^{\mathrm{f}}$ is the Coulomb potential due to $\rho_{\mathrm{f}}$. Converting the third term in eqn (29) to the atomic basis representation and taking its derivative with respect to $\xi$ leads to

$$
\begin{aligned}
\sum_{\mathrm{ia}, \mathrm{jb}} & (X+Y)_{\mathrm{ia}}(X+Y)_{\mathrm{jb}}\left[(\mathrm{ia} \mid \mathrm{jb})^{(\xi)}+f_{\mathrm{ia}, \mathrm{jb}}^{\mathrm{XC},(\xi)}+f_{\mathrm{XC}, \mathrm{ia}, \mathrm{jb}}^{\mathrm{nadd},(\xi)}+f_{\mathrm{kin}, \mathrm{ia}, \mathrm{jb}}^{\mathrm{nadd},(\xi)}\right] \\
= & \sum_{\mu \nu \kappa \lambda} \sum_{\mathrm{ia}, \mathrm{jb}}(X+Y)_{\mathrm{ia}}(X+Y)_{\mathrm{jb}} C_{\mu \mathrm{i}} C_{\mathrm{va}} C_{\mathrm{\kappa j}} C_{\lambda \mathrm{b}} \\
& \times\left[(\mu v \mid \kappa \lambda)^{\xi}+f_{\mu v, \kappa \lambda}^{\mathrm{XC},(\xi)}+f_{\mathrm{XC}, \mu v, \kappa \lambda}^{\mathrm{nadd},(\xi)}+f_{\mathrm{kin}, \mu v, \kappa \lambda}^{\mathrm{nadd},(\xi)}\right] \\
= & \sum_{\mu v \kappa \lambda}(X+Y)_{\mu \nu}(X+Y)_{\kappa \lambda} \\
& \times\left[(\mu \nu \mid \kappa \lambda)^{\xi}+f_{\mu \nu, \kappa \lambda}^{\mathrm{XC},(\xi)}+f_{\mathrm{XC}, \mu v, \kappa \lambda}^{\mathrm{nadd},(\xi)}+f_{\mathrm{kin}, \mu v, \kappa \lambda}^{\mathrm{nadd},(\xi)}\right],
\end{aligned}
$$

or, in terms of densities,

$$
\begin{aligned}
& =2 \int \rho^{(X+Y)(\xi)} v_{\mathrm{C}}^{(X+Y)}+2 \int \rho^{(X+Y)(\xi)} f^{\mathrm{XC}} \rho^{(X+Y)} \\
& \quad+2 \int \rho^{(X+Y)(\xi)} f_{\mathrm{XC}}^{\text {nadd }} \rho^{(X+Y)}+2 \int \rho^{(X+Y)(\xi)} f_{\mathrm{kin}}^{\mathrm{nadd}} \rho^{(X+Y)} \\
& \quad+\int \rho^{(X+Y)} \rho^{(X+Y)} \rho^{(\xi)} g^{\mathrm{XC}}+\int \rho^{(X+Y)} \rho^{(X+Y)} \rho^{(\xi)} g_{\mathrm{XC}}^{\text {nadd }} \\
& \quad+\int \rho^{(X+Y)} \rho^{(X+Y)} \rho^{(\xi)} g_{\text {kin }}^{\text {nadd }},
\end{aligned}
$$

where $g^{\mathrm{XC}}, g_{\mathrm{XC}}^{\text {nadd }}$, and $g_{\text {kin }}^{\text {nadd }}$ are given as functional derivatives of the kernels (third functional derivatives of the corresponding energy functionals with respect to the density).

The derivative of the last term in eqn (29) with respect to $\xi$ gives zero. Summing up all the terms mentioned above together with $\left(-\sum_{\mu \nu} S_{\mu \nu}^{\xi} W_{\mu \nu}\right)$ the expression for the Lagrangian derivative $L^{\xi}$ is

$$
\begin{aligned}
L^{\xi}= & T_{\mathrm{S}}\left[\rho^{\mathrm{P}(\xi)}\right] \\
& +\int \rho^{\mathrm{P}(\xi)}\left(v_{\mathrm{ext}}+v_{\mathrm{C}}+v^{\mathrm{XC}}+v_{\mathrm{ext}}^{\mathrm{f}}+v_{\mathrm{C}}^{\mathrm{f}}+v_{\mathrm{XC}}^{\text {nadd }}+v_{\mathrm{kin}}^{\text {nadd }}\right) \\
& +\int\left(v_{\mathrm{ext}}^{\xi} \rho^{\mathrm{P}}+v_{\mathrm{C}}^{\mathrm{P}} \rho^{(\xi)}\right)+\int \rho^{\mathrm{P}}\left(f^{\mathrm{XC}}+f_{\mathrm{XC}}^{\text {nadd }}+f_{\mathrm{kin}}^{\text {nadd }}\right) \rho^{(\xi)} \\
& +2 \int \rho^{(X+Y)(\xi)}\left[v_{\mathrm{C}}^{(X+Y)}+\left(f^{\mathrm{XC}}+f_{\mathrm{XC}}^{\text {nadd }}+f_{\text {kin }}^{\text {nadd }}\right) \rho^{(X+Y)}\right] \\
& +\int \rho^{(X+Y)} \rho^{(X+Y)} \rho^{(\xi)}\left[g^{\mathrm{XC}}+g_{\mathrm{XC}}^{\text {nadd }}+g_{\text {kin }}^{\text {nadd }}\right]-\sum_{\mu \nu} S_{\mu \nu}^{\xi} W_{\mu \nu} .
\end{aligned}
$$

\section{$2.4 Z$-vector equation for FDE-TDDFT}

For the evaluation of $L^{\xi}$, the corresponding vectors $\mathbf{W}$ and $\mathbf{Z}$ have to be known $(\mathbf{P}=\mathbf{Z}+\mathbf{T}$, and $\mathbf{T}$ is already determined from the solution of FDE-TDDFT problem). These vectors can be obtained from the so-called $Z$-vector equation, ${ }^{5,37}$ which is derived using the condition,

$$
\frac{\partial L}{\partial C_{\mu \mathrm{p}}}=0
$$

If eqn (44) is multiplied by $C_{\mu \mathrm{q}}$ and summed over index $\mu$, we get the following relation,

$$
Q_{\mathrm{pq}}+\sum_{\text {ia }} Z_{\mathrm{ia}} \sum_{\mu} \frac{\partial F_{\mathrm{ia}}}{\partial C_{\mu \mathrm{p}}} C_{\mu \mathrm{q}}=\sum_{\mathrm{rs}, \mathrm{r} \leq \mathrm{s}} W_{\mathrm{rs}} \sum_{\mu} \frac{\partial S_{\mathrm{rs}}}{\partial C_{\mu \mathrm{p}}} C_{\mu \mathrm{q}},
$$

where $Q$ stands for

$$
Q_{\mathrm{pq}}=\sum_{\mu} \frac{\partial G[\mathbf{X}, \mathbf{Y}, \omega]}{\partial C_{\mu \mathrm{p}}} C_{\mu \mathrm{q}}
$$

Introducing the abbreviation for some arbitrary $\mathbf{M}$,

$H_{\mathrm{ij}}^{+}[\mathbf{M}]=\sum_{\mathrm{rs}}\left[2(\mathrm{ij} \mid \mathrm{rs})+2 f_{\mathrm{ij}, \mathrm{rs}}^{\mathrm{XC}}+2 f_{\mathrm{XC}, \mathrm{ij}, \mathrm{rs}}^{\mathrm{nadd}}+2 f_{\mathrm{kin}, \mathrm{j}, \mathrm{rs}}^{\mathrm{nadd}}\right] M_{\mathrm{rs}}$,

it is possible to derive the following equations (see ref. 5 and Appendix B),

$$
\begin{gathered}
Q_{\mathrm{ij}}+H_{\mathrm{ij}}^{+}[\mathbf{Z}]=W_{\mathrm{ij}}\left(1+\delta_{\mathrm{ij}}\right), \\
Q_{\mathrm{ia}}+\varepsilon_{\mathrm{a}} Z_{\mathrm{ia}}+H_{\mathrm{ia}}^{+}[\mathbf{Z}]=W_{\mathrm{ia}}, \\
Q_{\mathrm{ai}}+\varepsilon_{\mathrm{i}} Z_{\mathrm{ia}}=W_{\mathrm{ia}}, \\
Q_{\mathrm{ab}}=W_{\mathrm{ab}}\left(1+\delta_{\mathrm{ab}}\right),
\end{gathered}
$$


where the explicit expressions for $Q_{\mathrm{pq}}$ look like (four cases are distinguished),

$$
\begin{aligned}
Q_{\mathrm{ij}}= & \sum_{\mathrm{a}} \omega\left[(X+Y)_{\mathrm{ia}}(X-Y)_{\mathrm{ja}}+(X-Y)_{\mathrm{ia}}(X+Y)_{\mathrm{ja}}\right] \\
& -\sum_{\mathrm{a}} \varepsilon_{\mathrm{a}}\left\{(X+Y)_{\mathrm{ia}}(X+Y)_{\mathrm{ja}}+(X-Y)_{\mathrm{ia}}(X-Y)_{\mathrm{ja}}\right\} \\
& +H_{\mathrm{ij}}^{+}[\mathbf{T}]+2 \sum_{\mathrm{kc}, \mathrm{ld}}(X+Y)_{\mathrm{kc}}\left[g^{\mathrm{XC}}+g_{\mathrm{XC}}^{\text {nadd }}+g_{\mathrm{kin}}^{\text {nadd }}\right]_{\mathrm{kc}, \mathrm{ld}, \mathrm{ij}} \\
& \times(X+Y)_{\mathrm{ld}}
\end{aligned}
$$$$
Q_{\mathrm{ia}}=\sum_{\mathrm{b}}(X+Y)_{\mathrm{ib}} H_{\mathrm{ab}}{ }^{+}[\mathbf{X}+\mathbf{Y}]+H_{\mathrm{ia}}{ }^{+}[\mathbf{T}]
$$$$
+2 \sum_{\mathrm{jb}, \mathrm{kc}}(X+Y)_{\mathrm{jb}}\left[g^{\mathrm{XC}}+g_{\mathrm{XC}}^{\mathrm{nadd}}+g_{\mathrm{kin}}^{\mathrm{nadd}}\right]_{\mathrm{jb}, \mathrm{kc}, \mathrm{ia}}(X+Y)_{\mathrm{kc}},
$$$$
Q_{\mathrm{ai}}=\sum_{\mathrm{j}}(X+Y)_{\mathrm{ja}} H_{\mathrm{ji}}{ }^{+}[\mathbf{X}+\mathbf{Y}]
$$$$
Q_{\mathrm{ab}}=\sum_{\mathrm{i}} \omega\left[(X+Y)_{\mathrm{ia}}(X-Y)_{\mathrm{ib}}+(X-Y)_{\mathrm{ia}}(X+Y)_{\mathrm{ib}}\right]
$$$$
+\sum_{\mathrm{i}} \varepsilon_{\mathrm{i}}\left\{(X+Y)_{\mathrm{ia}}(X+Y)_{\mathrm{ib}}+(X-Y)_{\mathrm{ia}}(X-Y)_{\mathrm{ib}}\right\} .
$$

Subtracting eqn (50) from eqn (49) we get the $Z$-vector equation

$$
\left(\varepsilon_{\mathrm{a}}-\varepsilon_{\mathrm{i}}\right) Z_{\mathrm{ia}}+H_{\mathrm{ia}}^{+}[\mathbf{Z}]=-\left(Q_{\mathrm{ia}}-Q_{\mathrm{ai}}\right) .
$$

This equation can be rewritten as

$$
\sum_{\mathrm{jb}}(A+B)_{\mathrm{ia}, \mathrm{jb}} Z_{\mathrm{jb}}=R_{\mathrm{ia}},
$$

where $R_{\mathrm{ia}}=-\left(Q_{\mathrm{ia}}-Q_{\mathrm{ai}}\right)$ reads

$$
\begin{aligned}
R_{\mathrm{ia}}= & -\left(\sum_{\mathrm{b}}\left\{(X+Y)_{\mathrm{ib}} H_{\mathrm{ab}}{ }^{+}[\mathbf{X}+\mathbf{Y}]\right\}\right. \\
& -\sum_{\mathrm{j}}\left\{(X+Y)_{\mathrm{ja}} H_{\mathrm{ji}}{ }^{+}[\mathbf{X}+\mathbf{Y}]\right\} \\
& +H_{\mathrm{ia}}{ }^{+}[\mathbf{T}]+2 \sum_{\mathrm{jb}, \mathrm{kc}}\left[g^{\mathrm{XC}}+g_{\mathrm{XC}}^{\text {nadd }}+g_{\mathrm{kin}}^{\text {nadd }}\right]_{\mathrm{jb}, \mathrm{kc}, \mathrm{ia}} \\
& \left.\times(X+Y)_{\mathrm{jb}}(X+Y)_{\mathrm{kc}}\right) .
\end{aligned}
$$

The matrix $\mathbf{W}$ is determined from the following equations

$$
\begin{gathered}
W_{\mathrm{ij}}=\frac{\left(Q_{\mathrm{ij}}+H_{\mathrm{ij}}^{+}[\mathbf{Z}]\right)}{\left(1+\delta_{\mathrm{ij}}\right)}, \\
W_{\mathrm{ab}}=\frac{Q_{\mathrm{ab}}}{\left(1+\delta_{\mathrm{ab}}\right)}, \\
W_{\mathrm{ia}}=Q_{\mathrm{ai}}+\varepsilon_{\mathrm{i}} Z_{\mathrm{ia}} .
\end{gathered}
$$

The $Z$-vector equation and all relevant variables can be rewritten in terms of densities. ${ }^{10}$ The corresponding quantities $H_{\mathrm{ij}}{ }^{+}[\mathbf{M}], Q_{\mathrm{ij}}$, and $Q_{\text {ia }}$ then read,

$$
\begin{aligned}
H_{\mathrm{ij}}{ }^{+}[\mathbf{M}] & =2 \int \phi_{\mathrm{i}} \phi_{\mathrm{j}}\left(v_{\mathrm{C}}^{\mathrm{M}}+\left[f^{\mathrm{XC}}+f_{\mathrm{XC}}^{\mathrm{nadd}}+f_{\mathrm{kin}}^{\mathrm{nadd}}\right] \rho^{\mathrm{M}}\right) \\
Q_{\mathrm{ij}}= & \sum_{\mathrm{a}} \omega\left[(X+Y)_{\mathrm{ia}}(X-Y)_{\mathrm{ja}}+(X-Y)_{\mathrm{ia}}(X+Y)_{\mathrm{ja}}\right] \\
& -\sum_{\mathrm{a}} \varepsilon_{\mathrm{a}}\left\{(X+Y)_{\mathrm{ia}}(X+Y)_{\mathrm{ja}}+(X-Y)_{\mathrm{ia}}(X-Y)_{\mathrm{ja}}\right\} \\
& +H_{\mathrm{ij}}{ }^{+}[\mathbf{T}]+2 \int \phi_{\mathrm{i}}\left[g^{\mathrm{XC}}+g_{\mathrm{XC}}^{\text {nadd }}+g_{\mathrm{kin}}^{\mathrm{nadd}}\right] \rho^{(X+Y)} \rho^{(X+Y)} \\
Q_{\mathrm{ia}}= & \sum_{\mathrm{b}}(X+Y)_{\mathrm{ib}} H_{\mathrm{ab}}{ }^{+}[\mathbf{X}+\mathbf{Y}]+H_{\mathrm{ia}}{ }^{+}[\mathbf{T}] \\
& +2 \int \phi_{\mathrm{i}}\left[g^{\mathrm{XC}}+g_{\mathrm{XC}}^{\mathrm{nadd}}+g_{\mathrm{kin}}^{\text {nadd }}\right] \phi_{\mathrm{a}} \rho^{(X+Y)} \rho^{(X+Y)}
\end{aligned}
$$

while $Q_{\mathrm{ai}}$ and $Q_{\mathrm{ab}}$ will remain the same. Note that $\rho^{\mathrm{M}}$ and $\rho^{(X+Y)}$ are defined in analogy to eqn (37). Finally, one can rewrite $R_{\mathrm{ia}}$ in terms of densities

$$
\begin{aligned}
R_{\mathrm{ia}}= & -\left(\sum_{\mathrm{b}}\left\{(X+Y)_{\mathrm{ib}} H_{\mathrm{ab}}{ }^{+}[\mathbf{X}+\mathbf{Y}]\right\}\right. \\
& -\sum_{\mathrm{j}}\left\{(X+Y)_{\mathrm{ja}} H_{\mathrm{ji}}{ }^{+}[\mathbf{X}+\mathbf{Y}]\right\}+{H_{\mathrm{ia}}}^{+}[\mathbf{T}] \\
& \left.+2 \int \phi_{\mathrm{i}}\left[g^{\mathrm{XC}}+g_{\mathrm{XC}}^{\text {nadd }}+g_{\text {kin }}^{\text {nadd }}\right] \phi_{\mathrm{a}} \rho^{(X+Y)} \rho^{(X+Y)}\right) .
\end{aligned}
$$

\section{Details of the implementation}

An algorithm for the evaluation of excitation-energy gradients within FDEu-TDDFT was implemented in ADF. ${ }^{11}$ Our implementation is based on the modules for the calculation of excitation-energy gradients in TDDFT, which were developed by Seth $e t$ al. ${ }^{10}$ In addition, we created modules for the evaluation of the second functional derivative of the non-additive kinetic energy using the AGGA (denoted as FULL in the formulas below to distinguish from the commonly used ALDA approximation), and the second and third functional derivatives of the non-additive kinetic energy using the Thomas Fermi approximation (denoted as $\mathrm{TF}$ in the formulas below). These modules make use of the XCFUN library. ${ }^{38}$ As was already mentioned in Section 2.2, the evaluation of the second functional derivative in response calculations is usually done within the ALDA in ADF, independently of the approximation used for the XC potential. This introduces some consistency issues in the evaluation of TDDFT gradients. Every kernel and potential term in the expression for the gradient which originates from the Fock matrix (XC potential and second XC functional derivative) have to be evaluated in the original approximation (LDA, GGA,...), while terms which result from the response part are always 
evaluated in the ALDA (which in the case of the non-additive kinetic energy is the TF approximation). Within the ADF setup, the ALDA-consistent expression for the excited-state gradients thus reads,

$$
\begin{aligned}
L^{\xi}= & T_{\mathrm{s}}\left[\rho^{\mathrm{P}(\xi)}\right] \\
& +\int \rho^{\mathrm{P}(\xi)}\left(v_{\mathrm{ext}}+v_{\mathrm{C}}+v^{\mathrm{XC}}+v_{\mathrm{ext}}^{\mathrm{f}}+v_{\mathrm{C}}^{\mathrm{f}}+v_{\mathrm{XC}}^{\mathrm{nadd}}+v_{\mathrm{kin}}^{\mathrm{nadd}}\right) \\
& +\int\left(v_{\mathrm{ext}}^{\xi} \rho^{\mathrm{P}}+v_{\mathrm{C}}^{\mathrm{P}} \rho^{(\xi)}\right) \\
& +\int \rho^{\mathrm{P}}\left(f_{\mathrm{FULL}}^{\mathrm{XC}}+f_{\mathrm{XC}, \mathrm{FULL}}^{\mathrm{nadd}}+f_{\mathrm{kin}, \mathrm{FULL}}^{\mathrm{nadd}}\right) \rho^{(\xi)} \\
& +2 \int \rho^{(X+Y)(\xi)}\left[v_{\mathrm{C}}^{(X+Y)}+\left(f_{\mathrm{ALDA}}^{\mathrm{XC}}+f_{\mathrm{XC}, \mathrm{ALDA}}^{\mathrm{nadd}}+f_{\mathrm{kin}, \mathrm{TF}}^{\mathrm{nadd}}\right) \rho^{(X+Y)}\right] \\
& +\int \rho^{(X+Y)} \rho^{(X+Y)} \rho^{(\xi)}\left[g_{\mathrm{ALDA}}^{\mathrm{XC}}+g_{\mathrm{XC}, \mathrm{ALDA}}^{\mathrm{nadd}}+g_{\mathrm{kin}, \mathrm{TF}}^{\mathrm{nadd}}\right] \\
& -\sum_{\mu \nu} S_{\mu \nu}^{\xi} W_{\mu \nu} .
\end{aligned}
$$

The difference in the approximations for the $\mathrm{XC}$ part has to be taken into account in the $Z$-vector equation, eqn (57), as well. Special attention should be paid to the elements $H_{\mathrm{ij}}{ }^{+}[\mathbf{M}]$. Note that

$$
H_{\mathrm{ij}}^{+}[\mathbf{M}]=2 \int \phi_{\mathrm{i}} \phi_{\mathrm{j}}\left(v_{\mathrm{C}}^{\mathrm{M}}+\left[f_{\mathrm{ALDA}}^{\mathrm{XC}}+f_{\mathrm{XC}, \mathrm{ALDA}}^{\mathrm{nadd}}+f_{\mathrm{kin}, \mathrm{TF}}^{\mathrm{nadd}}\right] \rho^{\mathrm{M}}\right),
$$

when $\mathbf{M}$ is $(\mathbf{X}+\mathbf{Y})$, and

$$
H_{\mathrm{ij}}{ }^{+}[\mathbf{M}]=2 \int \phi_{\mathrm{i}} \phi_{\mathrm{j}}\left(v_{\mathrm{C}}^{\mathrm{M}}+\left[f_{\mathrm{FULL}}^{\mathrm{XC}}+f_{\mathrm{XC}, \mathrm{FULL}}^{\mathrm{nadd}}+f_{\mathrm{kin}, \mathrm{FULL}}^{\mathrm{nadd}}\right] \rho^{\mathrm{M}}\right),
$$

when $\mathbf{M}$ is $\mathbf{T}$ or $\mathbf{Z}$.

An additional comment should be made on the evaluation of the non-additive XC kernels. One can significantly simplify the evaluation of the XC kernels and their functional derivatives if the expression for the non-additive XC potential, eqn (7) is recalled,

$$
f_{\mathrm{XC}}[\rho]+f_{\mathrm{XC}}^{\mathrm{nad}}\left[\rho_{\mathrm{tot}}, \rho\right]=f_{\mathrm{XC}}[\rho]+f_{\mathrm{XC}}\left[\rho_{\mathrm{tot}}\right]-f_{\mathrm{XC}}[\rho]=f_{\mathrm{XC}}\left[\rho_{\text {tot }}\right] \text {, }
$$

$$
g_{\mathrm{XC}}[\rho]+g_{\mathrm{XC}}^{\mathrm{nadd}}\left[\rho_{\mathrm{tot}}, \rho\right]=g_{\mathrm{XC}}[\rho]+g_{\mathrm{XC}}\left[\rho_{\mathrm{tot}}\right]-g_{\mathrm{XC}}[\rho]=g_{\mathrm{XC}}\left[\rho_{\text {tot }}\right] \text {. }
$$

This basically means that instead of the evaluation and summation of XC kernels for active and total densities, one needs to evaluate the XC kernels only for the total density $\rho_{\text {tot }}(\mathbf{r})$.

Concerning the computational scaling of the FDEu-TDDFT gradient calculations, we note that only a small additional step in the calculation of embedding potentials and kernels is needed, compared to the effort in the standard TDDFT gradients. In particular, the orbital space is not increased in FDEu-TDDFT calculations when using monomer basis sets for the active system (which is the default in these types of calculations).
The differences in timing and computational effort of the ground-state DFT step and in TDDFT excitation-energy calculations have been addressed in ref. 23, and we refer the interested reader to this reference for further details.

\section{Computational details}

A locally modified version of the ADF package ${ }^{11,12}$ was used for all calculations. For ground-state structure optimizations, the Becke-Perdew (BP86) XC functional ${ }^{39,40}$ in combination with the triple- $\zeta$ polarized Slater-type (TZP) basis set from the ADF basis set library was employed. The same basis set and the $\mathrm{XC}$ functional were used for the excitation energy calculations. For the FDE calculations, we employed the corresponding ADF implementation, ${ }^{41}$ making use of the so-called GGA97 generalized-gradient approximation (GGA) for the non-additive kinetic contribution to the embedding potential. ${ }^{42}$ This approximation is also known as PW91k, because it has the same functional form for the enhancement factor as used in the exchange functional of Perdew and Wang (PW91). ${ }^{43}$

\section{Results and discussions}

To test the analytical gradients for excitation energies within the FDEu approach, three different test systems were studied. A first test was performed on the complex of formaldehyde with a lithium ion (charge +1 ), where the density of the lithium ion was frozen. The structure of the system was obtained in the following way: at first, the formaldehyde structure was optimized with BP86/TZP; then the lithium ion was put on the axis along the CO bond (oriented along the $z$-axis). The structure is shown in Fig. 1. Such an orientation of the lithium ion was chosen to enforce a rather large effect of the embedding potential in this initial test. This system is thus highly polarized, and the effect of the classical electrostatic part of the embedding potential will be quite pronounced. But in addition, the nonadditive kinetic-energy contributions can be important in such systems, because they are necessary to prevent over-polarization effects.

The excitation energy dominated by the HOMO $\rightarrow$ LUMO transition was chosen for the calculation of the gradient at different $\mathrm{C}=\mathrm{O}$ bond distances. Note that the $\mathrm{C} \cdots \mathrm{Li}^{+}$distance is kept constant in our calculations, so that increasing $\mathrm{C}=\mathrm{O}$ distances correspond to decreasing $\mathrm{O} \cdots \mathrm{Li}^{+}$distances. The HOMO of this
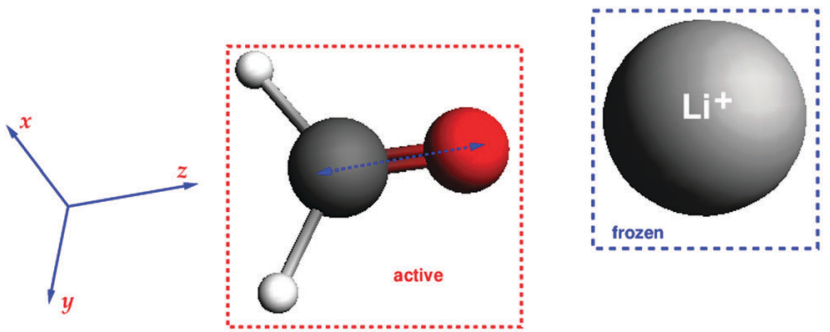

Fig. 1 Structure of the $\mathrm{CH}_{2} \mathrm{O} \cdots \mathrm{Li}^{+}$system and its orientation in coordinate space. 


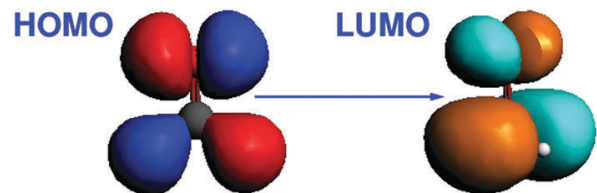

Fig. 2 Isosurface plots of the orbitals (BP86/TZP) involved in the dominant orbital transition of the excitation under study for $\mathrm{CH}_{2} \mathrm{O} \cdots \mathrm{Li}^{+}$(lithium ion is not shown).

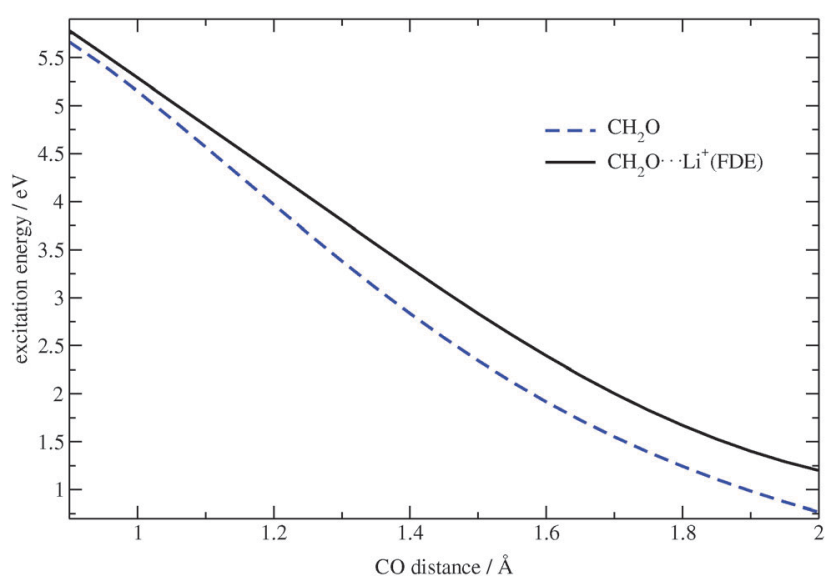

Fig. 3 Dependence of the excitation energy on the $\mathrm{C}=\mathrm{O}$ bond distance in $\mathrm{CH}_{2} \mathrm{O}$ and $\mathrm{CH}_{2} \mathrm{O} \cdots \mathrm{Li}^{+}$(TZP/BP86).

system corresponds to a nonbonding (n) orbital, while the LUMO corresponds to a $\pi^{*}$ orbital. Isosurface plots of the orbitals involved in the transition are shown in Fig. 2.

To check whether the embedding potential and all of its components affect the electronic transitions, the excitation energies for the $\mathrm{CH}_{2} \mathrm{O} \cdots \mathrm{Li}^{+}$complex and for isolated formaldehyde were calculated for different $\mathrm{C}=\mathrm{O}$ bond distances. These results are shown in Fig. 3. One can see from this figure that the closer the oxygen atom is to the lithium ion, the more the excitation energy is affected by the embedding potential. Thus, the complex represents a good test system to check the implemented procedure.

The gradients of the excitation energies ${ }^{10}$ for isolated formaldehyde for different $\mathrm{C}=\mathrm{O}$ bond distances were calculated analytically with BP86/TZP. Then for the same distances gradients were calculated numerically for the complex, where the lithium ion was described with FDEu. In addition, the analytical gradients of the excitation energy were calculated for the same system with FDEu. To compare the obtained results, the dependence of the $z$-component of the gradient for the oxygen atom on the $\mathrm{C}=\mathrm{O}$ bond distance is plotted for each calculation in Fig. 4 .

One can see from the plot that the presence of the lithium ion is simulated very well by the embedding potential, and that it contributes significantly to the value of the excitation-energy gradient. For all displacements of the oxygen atom towards lithium, the values of the $z$-component of the gradient for oxygen are in perfect agreement.

In the second test system, the lithium ion was substituted by a helium atom, and the $\mathrm{O} \cdots$ He distance was changed while

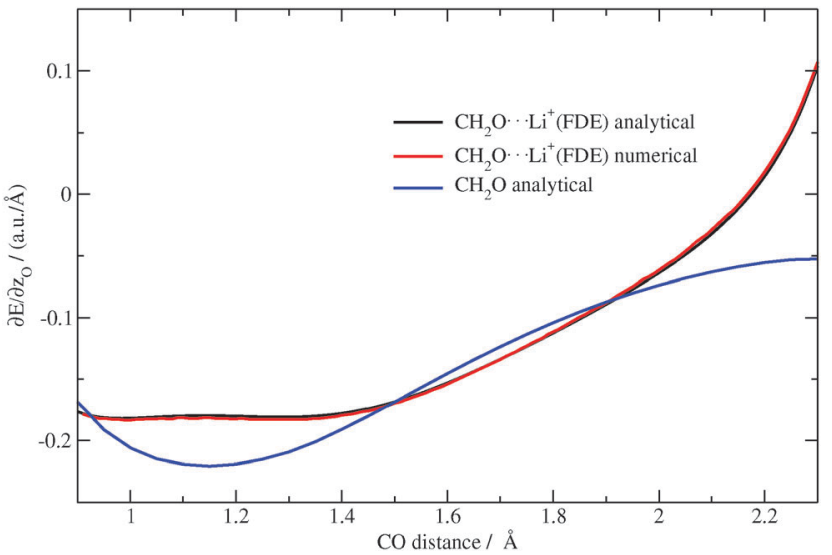

Fig. 4 Dependence of the $z$-component of the gradient of the excitation energy for the oxygen atom in $\mathrm{CH}_{2} \mathrm{O}$ (analytical) and $\mathrm{CH}_{2} \mathrm{O} \cdots \mathrm{Li}^{+}$(analytical/ numerical) on the $\mathrm{CO}$ distance (TZP/BP86/PW91K).

keeping the $\mathrm{C}=\mathrm{O}$ distance fixed at $1.7 \AA$. This is significantly larger than the ground-state equilibrium $\mathrm{C}=\mathrm{O}$ distance of about $1.2 \AA$, and was selected in order to observe a significant effect of the embedding contribution (at the equilibrium distance, this effect is rather small). Contrary to the first, the second test system has a small contribution of the classical electrostatic part to the embedding potential. The same excitation as in the previous case was considered, see Fig. 2. The effect of the FDE potential on the excitation energy under study can clearly be seen in Fig. 5.

Again, as in the previous case, the dependence of the $z$-component of the gradient for the oxygen atom on the $\mathrm{O} \cdots \mathrm{He}$ distance is plotted in Fig. 6 for the $\mathrm{CH}_{2} \mathrm{O}$ molecule alone as well as for the $\mathrm{CH}_{2} \mathrm{O} \cdots \mathrm{He}$ system (analytical/numerical). We observe a very good agreement between numerical and analytical results. The small remaining discrepancies can be attributed to inaccuracies of the numerical gradient. This can be seen by considering the region from $3.1 \AA$ to $3.3 \AA$ (see inset in Fig. 6). In this region the influence of the $\mathrm{He}$ atom on the gradient

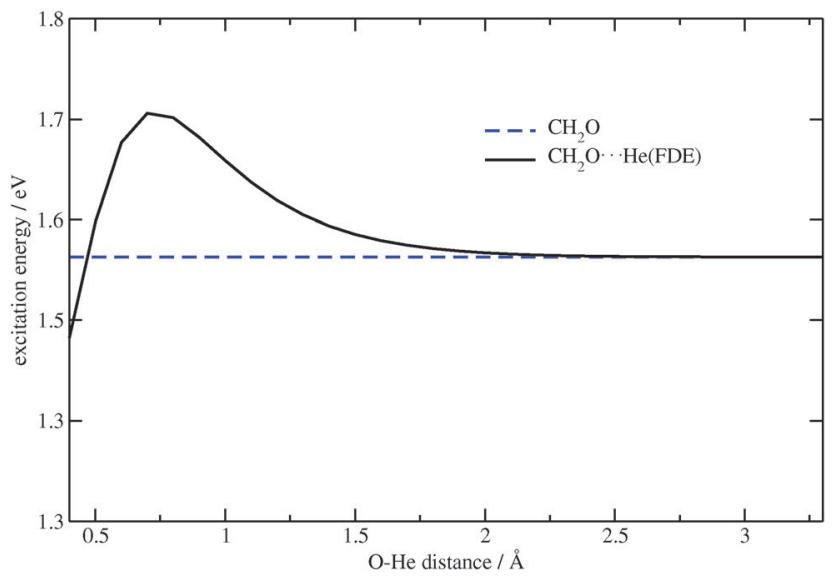

Fig. 5 Dependence of the excitation energy on the $\mathrm{O}$...He distance for $\mathrm{CH}_{2} \mathrm{O} \cdots \mathrm{He}$ (TZP/BP86). The excitation energy for $\mathrm{CH}_{2} \mathrm{O}$ (constant in this plot) is shown for comparison. 


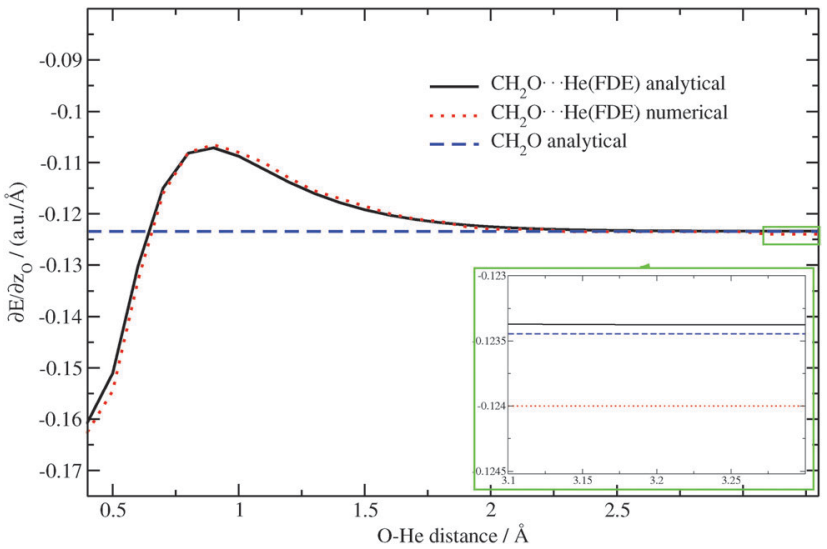

Fig. 6 Dependence of the $z$-component of the gradient of the excitation energy for the oxygen atom in $\mathrm{CH}_{2} \mathrm{O} \ldots \mathrm{He}$ (analytical/numerical) and in $\mathrm{CH}_{2} \mathrm{O}$ (analytical) on the O...He distance (TZP/BP86/PW91K). The region from $3.1 \AA$ to $3.3 \AA$ is magnified in the inset.

should vanish and the result should be very close to the one for the isolated molecule. While the analytical FDE gradient approaches the result for the isolated molecule with good accuracy, the numerical one underestimates the $z$-component of the gradient by roughly 0.0005 a.u. $\AA^{-1}$. This shows the advantages of the analytical approach over the numerical one. Note that the error for the numerical approach might be larger in regions where the gradient changes faster, e.g. near $0.4 \AA$, see Fig. 6 .

The third test system consists of an acetone molecule and water treated with FDE. We take this system from our previous work on frozen density embedding. ${ }^{44}$ The system was optimized with BP86/ TZP imposing $C_{\mathrm{s}}$ symmetry, see Fig. 7. Here, we studied the excitation dominated by the HOMO $\rightarrow$ LUMO $\left(\mathrm{n} \rightarrow \pi^{*}\right)$ orbital transition. The orbital composition of the excitation considered for the gradient calculation is shown in Fig. 8.

In comparison to the previous tests where only single atoms were considered as frozen systems within FDE, here we deal

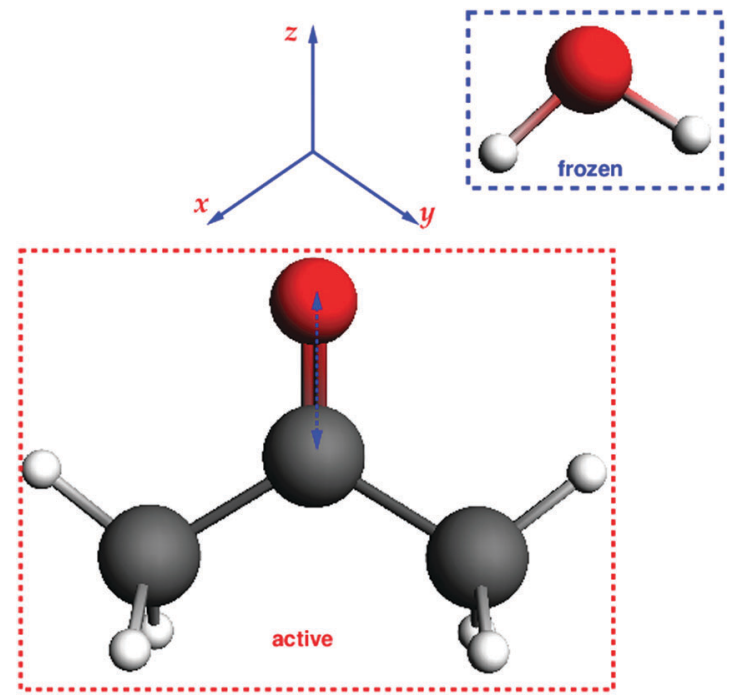

Fig. 7 Structure of the acetone...water system and its orientation in coordinate space.

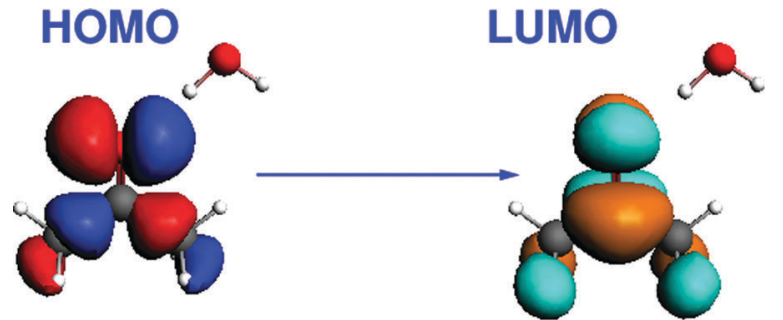

Fig. 8 Isosurface plots of the orbitals (BP86/TZP) involved in the dominant orbital transition of the excitation studied for the acetone...water system.

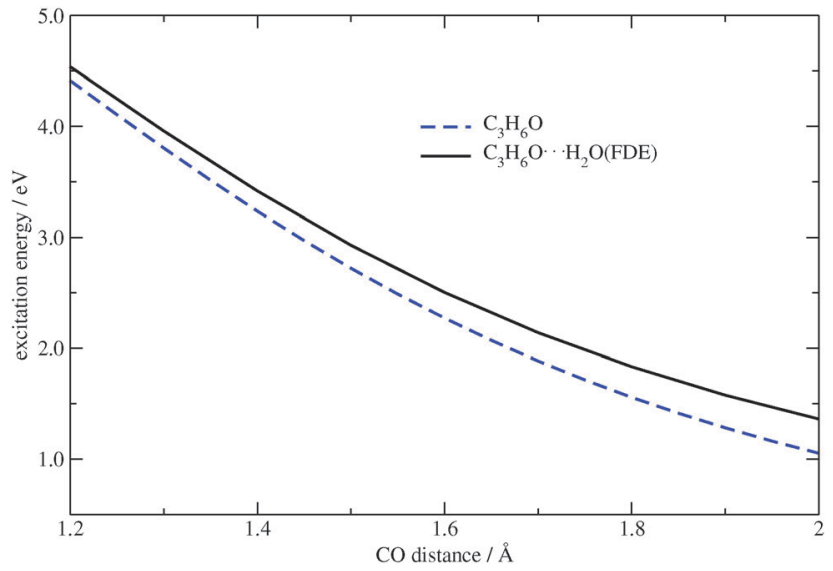

Fig. 9 Dependence of the excitation energy on the $\mathrm{CO}$ distance in $\mathrm{C}_{3} \mathrm{H}_{6} \mathrm{O}$ and $\mathrm{C}_{3} \mathrm{H}_{6} \mathrm{O} \cdots \mathrm{H}_{2} \mathrm{O}$ (TZP/BP86).

with a frozen molecule. In this case, both electrostatic and nonadditive kinetic parts of the FDE potential are significant. Structures in which the $\mathrm{C}=\mathrm{O}$ bond distance in acetone was varied from $1.2 \AA$ to $2 \AA$ were generated for the comparison of numerical and analytical gradients. Similar to our first test system, the carbon-water distance was kept fixed, i.e., longer $\mathrm{C}=\mathrm{O}$ distances correspond to shorter $\mathrm{O} \cdots \mathrm{H}_{2} \mathrm{O}$ contacts. In Fig. 9 the dependence of the excitation energy on the $\mathrm{C}=\mathrm{O}$

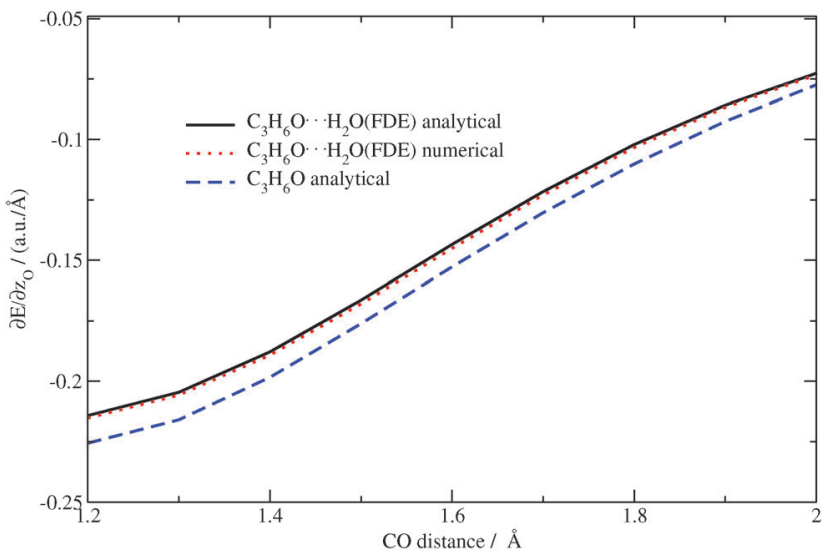

Fig. 10 Dependence of the $z$-component of the gradient of the excitation energy for the (acetone-)oxygen atom in the acetone... water system on the CO distance in acetone (TZP/BP86/PW91K). 
bond distance for isolated acetone and for the acetone $\cdots$ water system are plotted. One can see that the FDE potential has a slight effect on the excitation energy.

The analytical and numerical excitation energy gradients for $\mathrm{C}_{3} \mathrm{H}_{6} \mathrm{O} \cdots \mathrm{H}_{2} \mathrm{O}$ as well as for isolated acetone were calculated for the above-mentioned structures. The $z$-component of the excitation energy gradient for the oxygen atom in acetone (analytical) and in acetone - -water (analytical/numerical) are shown in Fig. 10. Again, the results from the analytical approach are in good agreement with the numerical ones.

\section{Summary and conclusions}

In this paper we have, for the first time, presented the formulation of analytical excitation-energy gradients in FDE-TDDFT. Furthermore, an algorithm and an implementation for the analytical evaluation of excitation-energy gradients within the FDEu approximation were developed and discussed in detail. We have shown how to incorporate the contributions from the embedding potential into the final expression for excitationenergy gradients consistently with the non-FDE implementation in $\mathrm{ADF}$ by Seth et $a{ }^{10}{ }^{10}$ Special care has to be taken concerning approximations for non-additive kinetic and $\mathrm{XC}$ kernel and their functional derivatives in order to be consistent with the previous ALDA implementation. The kernels arising from the Fock operator should be evaluated in the original approximation, while terms originating from the response part are evaluated in the ALDA. Of course, the implementation is flexible and allows for an extension beyond the ALDA in future work.

The implemented procedure was successfully tested and yields very good agreement with numerical reference results. Three different test systems were considered, which were chosen according to the expected contribution of different parts of the FDE embedding potential. In particular, the correct behavior of the gradient was tested on formaldehyde with a lithium ion as the frozen part and on formaldehyde with a frozen helium atom. The former has a large contribution from classical electrostatics in the FDE potential, while in the latter system the contribution from the non-additive kinetic potential is more important. As another test with a frozen molecular environment system, an acetone.-.water complex was chosen. All calculations on the above-mentioned systems showed good agreement between analytical and numerical results. Moreover, it was demonstrated that results of analytical FDEu-TDDFT gradients are more accurate and thus preferable to the ones obtained by numerical differentiation.

The whole formalism was successfully implemented into the ADF package. In future work, we plan to address more complicated test systems to optimize the performance of the implementation and make FDE calculations beyond the ALDA possible. Also extensions beyond the approximation of a response localized on the active system are desirable if subsystem-based descriptions of excitonically coupled chromophores ${ }^{45}$ or explicit solvent response effects ${ }^{46}$ are of interest.

\section{A Detailed derivation of the excitation- energy gradients}

\section{A.1 Introduction}

In this appendix, we provide an in-depth derivation of the expression for excitation-energy gradients in FDE-TDDFT, based on the studies of Furche and Ahlrichs ${ }^{5}$ as well as Seth et $a{ }^{10}{ }^{10}$ The latter directly focuses on aspects relevant for the Slater-based ADF framework and thus provides the basis for the derivation of excited-state gradients in FDE-TDDFT, which is established here in Sections 2.3 and 2.4.

\section{A.2 Excited state gradients for TDDFT}

In FDE-TDDFT, the excited-state energy is represented as a sum of the ground-state energy and the excitation energy. If the gradient of the excited-state energy has to be evaluated, one has to consider the derivatives of these two terms. The derivatives of the former are formulated in ref. 31, and the derivative of the latter can be established in a similar way using Lagrangian techniques. We will only be concerned with the latter here. The variational formulation of FDE-TDDFT (by analogy to TDDFT case $^{5,47,48}$ ) reads

$$
\begin{aligned}
& \frac{\partial G[\mathbf{X}, \mathbf{Y}, \omega]}{\partial\langle\mathbf{X}, \mathbf{Y}|}=0, \\
& \frac{\partial G[\mathbf{X}, \mathbf{Y}, \omega]}{\partial \omega}=0,
\end{aligned}
$$

where the Lagrangian $G[\mathbf{X}, \mathbf{Y}, \omega]$ for the excitation energy has the following form

$$
G[\mathbf{X}, \mathbf{Y}, \omega]=\langle\mathbf{X}, \mathbf{Y}|\Lambda| \mathbf{X}, \mathbf{Y}\rangle-\omega(\langle\mathbf{X}, \mathbf{Y}|\Delta| \mathbf{X}, \mathbf{Y}\rangle-1) .
$$

The quantities $\Lambda$ and $\Delta$ are super-matrices,

$$
\begin{aligned}
& \Lambda=\left[\begin{array}{ll}
\mathbf{A} & \mathbf{B} \\
\mathbf{B} & \mathbf{A}
\end{array}\right], \\
& \Delta=\left[\begin{array}{cc}
\mathbf{1} & \mathbf{0} \\
\mathbf{0} & -\mathbf{1}
\end{array}\right] .
\end{aligned}
$$

The $|\mathbf{X}, \mathbf{Y}\rangle$ vector in Dirac notation is

$$
|\mathbf{X}, \mathbf{Y}\rangle=\left[\begin{array}{l}
\mathbf{X} \\
\mathbf{Y}
\end{array}\right]
$$

After differentiation and elementary manipulations, eqn (71) leads to a set of two matrix-vector equations, eqn (23) and (24) given in Section 2.2. The first term of the Lagrangian in eqn (73), $\langle\mathbf{X}, \mathbf{Y}|\Lambda| \mathbf{X}, \mathbf{Y}\rangle$, can be rewritten in the following way,

$$
\begin{aligned}
{\left[\mathbf{X}^{\dagger} \mathbf{Y}^{\dagger}\right]\left[\begin{array}{cc}
\mathbf{A} & \mathbf{B} \\
\mathbf{B} & \mathbf{A}
\end{array}\right]\left[\begin{array}{l}
\mathbf{X} \\
\mathbf{Y}
\end{array}\right]=} & \mathbf{X}^{\dagger} \mathbf{A} \mathbf{X}+\mathbf{Y}^{\dagger} \mathbf{B} \mathbf{X}+\mathbf{X}^{\dagger} \mathbf{B Y}+\mathbf{Y}^{\dagger} \mathbf{A} \mathbf{Y} \\
= & \frac{1}{2} \sum_{\mathrm{ia}, \mathrm{jb}}\left[(A+B)_{\mathrm{ia}, \mathrm{jb}}(X+Y)_{\mathrm{ia}}(X+Y)_{\mathrm{jb}}\right. \\
& \left.+(A-B)_{\mathrm{ia}, \mathrm{jb}}(X-Y)_{\mathrm{ia}}(X-Y)_{\mathrm{jb}}\right] .
\end{aligned}
$$


After substitution of this result into eqn (73) and writing the second term in an explicit way, the Lagrangian will take the following form

$$
\begin{aligned}
G[\mathbf{X}, \mathbf{Y}, \omega]= & \frac{1}{2} \sum_{\mathrm{ia}, \mathrm{jb}}\left[(A+B)_{\mathrm{ia}, \mathrm{jb}}(X+Y)_{\mathrm{ia}}(X+Y)_{\mathrm{jb}}\right. \\
& \left.+(A-B)_{\mathrm{ia}, \mathrm{jb}}(X-Y)_{\mathrm{ia}}(X-Y)_{\mathrm{jb}}\right] \\
& -\omega\left[\sum_{\mathrm{ia}}(X+Y)_{\mathrm{ia}}(X-Y)_{\mathrm{ia}}-1\right] .
\end{aligned}
$$

If $G[\mathbf{X}, \mathbf{Y}, \omega]$ is expressed in this way, then the requirement of eqn (71) can equivalently be expressed in terms of two conditions $^{48}$

$$
\begin{aligned}
& \frac{\partial G[\mathbf{X}, \mathbf{Y}, \omega]}{\partial(X+Y)_{\mathrm{ia}}}=0, \\
& \frac{\partial G[\mathbf{X}, \mathbf{Y}, \omega]}{\partial(X-Y)_{\mathrm{ia}}}=0,
\end{aligned}
$$

for every combination ia, since one can use $(X+Y)_{\text {ia }}$ and $(X-Y)_{\text {ia }}$ instead of $X_{\mathrm{ia}}$ and $Y_{\mathrm{ia}}$ as free variables. These two equations lead to eqn (23) and (24). If the TDDFT eigenvalue problem is solved, then the Lagrangian is stationary with respect to $(X+Y)_{\text {ia }}$ and $(X-Y)_{\text {ia }}$ but not with respect to the molecular orbital coefficients $C_{\mu \mathrm{p}}$. This introduces difficulties if one studies the derivative of the Lagrangian with respect to some perturbation $\xi$,

$$
\begin{aligned}
\frac{\mathrm{d} G[\mathbf{X}, \mathbf{Y}, \omega]}{\mathrm{d} \xi}= & \sum_{\mu \mathrm{p}} \frac{\partial G[\mathbf{X}, \mathbf{Y}, \omega]}{\partial C_{\mu \mathrm{p}}} \frac{\partial C_{\mu \mathrm{p}}}{\partial \xi}+\sum_{\mathrm{ia}} \frac{\partial G[\mathbf{X}, \mathbf{Y}, \omega]}{\partial(X+Y)_{\mathrm{ia}}} \frac{\partial(X+Y)_{\mathrm{ia}}}{\partial \xi} \\
& +\sum_{\mathrm{ia}} \frac{\partial G[\mathbf{X}, \mathbf{Y}, \omega]}{\partial(X-Y)_{\mathrm{ia}}} \frac{\partial(X-Y)_{\mathrm{ia}}}{\partial \xi}+\frac{\partial G[\mathbf{X}, \mathbf{Y}, \omega]}{\partial \omega} \frac{\partial \omega}{\partial \xi} \\
& +\frac{\partial G[\mathbf{X}, \mathbf{Y}, \omega]}{\partial \xi} .
\end{aligned}
$$

The implicit dependence of $G[\mathbf{X}, \mathbf{Y}, \omega]$ on $\xi$ through $(X+Y)_{\text {ia }}$, $(X-Y)_{\text {ia }}$, and $\omega$ can be dropped using the conditions from eqn (72), (79) and (80). Thus, the derivative of $G[\mathbf{X}, \mathbf{Y}, \omega]$ can be simplified to

$$
\frac{\mathrm{d} G[\mathbf{X}, \mathbf{Y}, \omega]}{\mathrm{d} \xi}=\sum_{\mu \mathrm{p}} \frac{\partial G[\mathbf{X}, \mathbf{Y}, \omega]}{\partial C_{\mu \mathrm{p}}} \frac{\partial C_{\mu \mathrm{p}}}{\partial \xi}+\frac{\partial G[\mathbf{X}, \mathbf{Y}, \omega]}{\partial \xi} .
$$

The derivative of $G[\mathbf{X}, \mathbf{Y}, \omega]$ with respect to molecular orbital coefficients has the following form,

$$
\begin{aligned}
\frac{\partial G[\mathbf{X}, \mathbf{Y}, \omega]}{\partial C_{\mu \mathrm{p}}}= & \frac{1}{2} \sum_{\mathrm{ia}, \mathrm{jb}}\left[(X+Y)_{\mathrm{ia}}(X+Y)_{\mathrm{jb}} \frac{\partial(A+B)_{\mathrm{ia}, \mathrm{jb}}}{\partial C_{\mu \mathrm{p}}}\right. \\
& \left.+(X-Y)_{\mathrm{ia}}(X-Y)_{\mathrm{jb}} \frac{\partial(A-B)_{\mathrm{ia}, \mathrm{jb}}}{\partial C_{\mu \mathrm{p}}}\right] .
\end{aligned}
$$

Since

$$
\sum_{\mu \mathrm{p}} \frac{\partial G[\mathbf{X}, \mathbf{Y}, \omega]}{\partial C_{\mu \mathrm{p}}} \neq 0
$$

or, in other words, since the orbitals in the excited state are unrelaxed, the implicit dependence of $G[\mathbf{X}, \mathbf{Y}, \omega]$ on $\xi$ through the molecular orbital coefficients $C_{\mu \mathrm{p}}$ cannot be dropped. Thus the derivatives $\partial C_{\mu \mathrm{p}} / \partial \xi$ must be evaluated, which requires the solution of the $3 M$ Coupled-Perturbed Kohn-Sham (CPKS) equations if gradients shall be calculated ( $M$ is the number of atoms). These equations can be derived from the relation

$$
\frac{\partial F_{\mathrm{pq}}}{\partial \xi}=0,
$$

substituting $\xi$ with each coordinate of every atom in the system. This is a rather inefficient way to calculate the gradient of the excitation energy. But in 1984, Handy and Schaefer ${ }^{37}$ suggested an efficient method to avoid solving these $3 M$ equations, which instead requires to solve only one equation with the same dimension as the CPKS equation. This approach is called the $Z$-vector method ${ }^{49}$ and it is set up in such a way that it does not require derivatives of molecular orbital coefficients. ${ }^{5,37}$ It introduces the so-called relaxed difference density matrices ${ }^{5} \mathbf{P}$ (discussed in detail in Section A.6) and removes any implicit dependence of the Lagrangian on the molecular orbital coefficients. This can be done by introducing a new Lagrangian for the calculation of excitation energies in FDE-TDDFT (in analogy to $\mathrm{TDDFT}^{5}$ ),

$$
\begin{aligned}
L[\mathbf{X}, \mathbf{Y}, \omega, \mathbf{C}, \mathbf{Z}, \mathbf{W}]= & G[\mathbf{X}, \mathbf{Y}, \omega]+\sum_{\mathrm{ia}} Z_{\mathrm{ia}} F_{\mathrm{ia}} \\
& -\sum_{\mathrm{pq}, \mathrm{p} \leq \mathrm{q}} W_{\mathrm{pq}}\left(S_{\mathrm{pq}}-\delta_{\mathrm{pq}}\right),
\end{aligned}
$$

and requiring it to be stationary with respect to the all molecular orbital coefficients $C_{\mu \mathrm{p}}$ (for simplicity of notation the Lagrangian, $L[\mathbf{X}, \mathbf{Y}, \omega, \mathbf{C}, \mathbf{Z}, \mathbf{W}]$, will be written from now on as $L$ ),

$$
\frac{\partial L}{\partial C_{\mu \mathrm{p}}}=0
$$

The Lagrangian multipliers $Z_{\mathrm{ia}}$ and $W_{\mathrm{pq}}$ ensure implicit relaxation of the ground-state density and also enforce the relaxed density to be a solution of the KS equation. In particular, $\sum_{\mathrm{ia}} Z_{\mathrm{ia}} F_{\text {ia }}$ takes care of the conditions $F_{\text {ia }}=0$ (which implies that the KS equations are solved),

$$
\frac{\partial L}{\partial Z_{\mathrm{ia}}}=F_{\mathrm{ia}}=0
$$

and $\sum_{\mathrm{pq}, \mathrm{p} \leq \mathrm{q}} W_{\mathrm{pq}}\left(S_{\mathrm{pq}}-\delta_{\mathrm{pq}}\right)$ ensures orthonormality of the orbitals,

$$
\frac{\partial L}{\partial W_{\mathrm{pq}}}=\left(S_{\mathrm{pq}}-\delta_{\mathrm{pq}}\right)=0 .
$$


The relations below require that the Lagrangian satisfies the TDDFT eigenvalue problem

$$
\begin{gathered}
\frac{\partial L}{\partial\langle\mathbf{X}, \mathbf{Y}|}=\frac{\partial G}{\partial\langle\mathbf{X}, \mathbf{Y}|}=0, \\
\frac{\partial L}{\partial \omega}=\frac{\partial G}{\partial \omega}=0,
\end{gathered}
$$

where eqn (90) is the usual TDDFT eigenvalue problem and eqn (91) is the orthonormality constraint for the eigenvectors $|\mathbf{X}, \mathbf{Y}\rangle$.

\section{A.3 Expression for the Lagrangian in terms of MO integrals}

Substituting the results of eqn (13) and (14) into eqn (78), the expression for $G[\mathbf{X}, \mathbf{Y}, \omega]$ reads

$$
\begin{aligned}
G[\mathbf{X}, \mathbf{Y}, \omega]= & \frac{1}{2} \sum_{\mathrm{ia}, \mathrm{jb}}\left[(A+B)_{\mathrm{ia}, \mathrm{jb}}(X+Y)_{\mathrm{ia}}(X+Y)_{\mathrm{jb}}\right. \\
& \left.+(A-B)_{\mathrm{ia}, \mathrm{jb}}(X-Y)_{\mathrm{ia}}(X-Y)_{\mathrm{jb}}\right] \\
& -\omega \sum_{\mathrm{ia}}(X+Y)_{\mathrm{ia}}(X-Y)_{\mathrm{ia}} \\
= & \frac{1}{2} \sum_{\mathrm{ia}, \mathrm{jb}}\left[(X+Y)_{\mathrm{ia}}(X+Y)_{\mathrm{jb}}+(X-Y)_{\mathrm{ia}}(X-Y)_{\mathrm{jb}}\right] \\
& \times\left(F_{\mathrm{ab}} \delta_{\mathrm{ij}}-F_{\mathrm{ij}} \delta_{\mathrm{ab}}\right) \\
& +\frac{1}{2} \sum_{\mathrm{ia}, \mathrm{jb}}(X+Y)_{\mathrm{ia}}(X+Y)_{\mathrm{jb}} \\
& \times\left[2(\mathrm{ia} \mid \mathrm{jb})+2 f_{\mathrm{ia}, \mathrm{jb}}^{\mathrm{XC}}+2 f_{\mathrm{XC}, \mathrm{ia}, \mathrm{jb}}^{\mathrm{nadd}}+2 f_{\mathrm{kin}, \mathrm{ia}, \mathrm{jb}}^{\mathrm{nadd}}\right] \\
& -\omega\left[\sum_{\mathrm{ia}}(X+Y)_{\mathrm{ia}}(X-Y)_{\mathrm{ia}}-1\right] .
\end{aligned}
$$

For ease of notation the unrelaxed difference density matrix $\mathbf{T}$ is introduced,

$$
\begin{aligned}
& T_{\mathrm{ab}}=\frac{1}{2} \sum_{\mathrm{i}}\left\{(X+Y)_{\mathrm{ia}}(X+Y)_{\mathrm{ib}}+(X-Y)_{\mathrm{ia}}(X-Y)_{\mathrm{ib}}\right\}, \\
& T_{\mathrm{ij}}=-\frac{1}{2} \sum_{\mathrm{a}}\left\{(X+Y)_{\mathrm{ia}}(X+Y)_{\mathrm{ja}}+(X-Y)_{\mathrm{ia}}(X-Y)_{\mathrm{ja}}\right\},
\end{aligned}
$$

$$
T_{\mathrm{ia}}=T_{\mathrm{ai}}=0,
$$

where the last equality holds due to the properties of $(\mathbf{X}+\mathbf{Y})$ and $(\mathbf{X}-\mathbf{Y})$,

$$
\begin{aligned}
& (X+Y)_{\mathrm{ab}}=(X+Y)_{\mathrm{ij}}=0, \\
& (X-Y)_{\mathrm{ab}}=(X-Y)_{\mathrm{ij}}=0
\end{aligned}
$$

In eqn (92) the sum of terms containing $F_{\mathrm{ab}}$ over two indexes $\mathrm{i}, \mathrm{j}$ collapses to a sum over one index i because of $\delta_{\mathrm{ij}}$, which is then absorbed into $T_{\mathrm{ab}}$, see eqn (93). In the same way, the sum of terms containing $F_{\mathrm{ij}}$ over two indexes a, b collapses to a sum over one index a (because of $\delta_{\mathrm{ab}}$ ), which is included in the definition of $T_{\mathrm{ij}}$, see eqn (94). Considering eqn (95), the terms with $F_{\mathrm{ij}}$ and $F_{\mathrm{ab}}$ can be unified as terms with $F_{\mathrm{pq}}\left(\right.$ since $F_{\mathrm{ia}}=F_{\mathrm{ai}}=0$ ) and eqn (92) can be simplified to

$$
\begin{aligned}
G[\mathbf{X}, \mathbf{Y}, \omega]= & \sum_{\mathrm{pq}} T_{\mathrm{pq}} F_{\mathrm{pq}}+\sum_{\mathrm{ia}, \mathrm{jb}}(X+Y)_{\mathrm{ia}}(X+Y)_{\mathrm{jb}} \\
& \times\left[(\mathrm{ia} \mid \mathrm{jb})+f_{\mathrm{ia}, \mathrm{jb}}^{\mathrm{XC}}+f_{\mathrm{XC}, \mathrm{ia}, \mathrm{jb}}^{\text {nadd }}+f_{\text {kin }, \mathrm{ia}, \mathrm{jb}}^{\text {nadd }}\right] \\
& -\omega\left[\sum_{\mathrm{ia}}(X+Y)_{\mathrm{ia}}(X-Y)_{\mathrm{ia}}-1\right] .
\end{aligned}
$$

Using this form of $G[\mathbf{X}, \mathbf{Y}, \omega]$, eqn (86) takes the form,

$$
\begin{aligned}
L= & \sum_{\mathrm{pq}} T_{\mathrm{pq}} F_{\mathrm{pq}} \\
& +\sum_{\mathrm{ia}, \mathrm{jb}}(X+Y)_{\mathrm{ia}}(X+Y)_{\mathrm{jb}}\left[(\mathrm{ia} \mid \mathrm{jb})+f_{\mathrm{ia}, \mathrm{jb}}^{\mathrm{XC}}+f_{\mathrm{XC}, \mathrm{ia}, \mathrm{jb}}^{\mathrm{nadd}}+f_{\mathrm{kin}, \mathrm{ia}, \mathrm{jb}}^{\mathrm{nadd}}\right] \\
& -\omega\left[\sum_{\mathrm{ia}}(X+Y)_{\mathrm{ia}}(X-Y)_{\mathrm{ia}}-1\right] \\
& +\sum_{\mathrm{ia}} Z_{\mathrm{ia}} F_{\mathrm{ia}}-\sum_{\mathrm{pq}, \mathrm{p} \leq \mathrm{q}} W_{\mathrm{pq}}\left(S_{\mathrm{pq}}-\delta_{\mathrm{pq}}\right) .
\end{aligned}
$$

After the introduction of the relaxed difference density matrix $\mathbf{P}=\mathbf{T}+\mathbf{Z}$, the Lagrangian can be rewritten as

$$
\begin{aligned}
L= & \sum_{\mathrm{pq}} F_{\mathrm{pq}} P_{\mathrm{pq}} \\
& +\sum_{\mathrm{ia}, \mathrm{jb}}(X+Y)_{\mathrm{ia}}(X+Y)_{\mathrm{jb}}\left[(\mathrm{ia} \mid \mathrm{jb})+f_{\mathrm{ia}, \mathrm{jb}}^{\mathrm{XC}}+f_{\mathrm{XC}, \mathrm{i} a, \mathrm{jb}}^{\mathrm{nadd}}+f_{\text {kin }, \mathrm{i}, \mathrm{jb}}^{\mathrm{nadd}}\right] \\
& -\sum_{\mathrm{pq}, \mathrm{p} \leq \mathrm{q}}\left(S_{\mathrm{pq}}-\delta_{\mathrm{pq}}\right) W_{\mathrm{pq}}-\omega\left[\sum_{\mathrm{ia}}(X+Y)_{\mathrm{ia}}(X-Y)_{\mathrm{ia}}-1\right] .
\end{aligned}
$$

Recalling the Fock matrix, eqn (9), and using the definition of the core hamiltonian $\hat{h}=-\frac{1}{2} \nabla^{2}+v_{\text {ext }}(\mathbf{r})$, we get

$$
F_{\mathrm{pq}}=h_{\mathrm{pq}}+\sum_{\mathrm{i}}^{\mathrm{occ}}(\mathrm{pq} \mid \mathrm{ii})+v_{\mathrm{pq}}^{\mathrm{XC}}+\underbrace{v_{\mathrm{ext}, \mathrm{pq}}^{\mathrm{f}}+v_{\mathrm{C}, \mathrm{pq}}^{\mathrm{f}}+v_{\mathrm{XC}, \mathrm{pq}}^{\text {nadd }}+v_{\mathrm{kin}, \mathrm{pq}}^{\text {nadd }}}_{v_{\mathrm{pq}}^{\mathrm{mb}}},
$$


and we can finally transform the Lagrangian to the following form,

$$
\begin{aligned}
L= & \sum_{\mathrm{pq}} h_{\mathrm{pq}} P_{\mathrm{pq}}+\sum_{\mathrm{pq}} \sum_{\mathrm{i}}^{\mathrm{occ}}(\mathrm{pq} \mid \mathrm{ii}) P_{\mathrm{pq}}+\sum_{\mathrm{pq}} P_{\mathrm{pq}}\left(v_{\mathrm{pq}}^{\mathrm{XC}}+v_{\mathrm{pq}}^{\mathrm{emb}}\right) \\
& +\sum_{\mathrm{ia}, \mathrm{jb}}(X+Y)_{\mathrm{ia}}(X+Y)_{\mathrm{jb}} \\
& \times\left[(\mathrm{ia} \mid \mathrm{jb})+f_{\mathrm{ia}, \mathrm{jb}}^{\mathrm{XC}}+f_{\mathrm{XC}, \mathrm{ia}, \mathrm{jb}}^{\mathrm{nadd}}+f_{\mathrm{kin}, \mathrm{ia}, \mathrm{jb}}^{\mathrm{nadd}}\right] \\
& -\sum_{\mathrm{pq}, \mathrm{p} \leq \mathrm{q}}\left(S_{\mathrm{pq}}-\delta_{\mathrm{pq}}\right) W_{\mathrm{pq}}-\omega\left[\sum_{\mathrm{ia}}(X+Y)_{\mathrm{ia}}(X-Y)_{\mathrm{ia}}-1\right] .
\end{aligned}
$$

\section{A.4 Expression for the Lagrangian in terms of AO integrals}

The imposed requirement for the Lagrangian to be stationary with respect to the elements of $\mathbf{C}$,

$$
\frac{\partial L}{\partial C_{\mu p}}=0
$$

cancels out the implicit dependence of $L$ on a perturbation $\xi$ through the MO coefficients $C_{\mu \mathrm{p}}$. Hence it is more convenient to convert all terms to atomic orbitals by means of the relation,

$$
\phi_{\mathrm{p}}=\sum_{\mu} C_{\mu \mathrm{p}} \chi_{\mu}
$$

Noting that

$$
h_{\mathrm{pq}}=\sum_{\mu v} C_{\mu \mathrm{p}} C_{\mathrm{vq}} h_{\mu v}
$$

and defining,

$$
P_{\mu v}:=\sum_{\mathrm{pq}} C_{\mu \mathrm{p}} C_{\mathrm{vq}} P_{\mathrm{pq}}
$$

the first term in eqn (102) results in,

$$
\sum_{\mathrm{pq}} h_{\mathrm{pq}} P_{\mathrm{pq}}=\sum_{\mathrm{pq}} \sum_{\mu v} h_{\mu \nu} C_{\mu \mathrm{p}} C_{\mathrm{vq}} P_{\mathrm{pq}}=\sum_{\mu \nu} h_{\mu v} P_{\mu v}
$$

From the second term, we obtain,

$$
\begin{aligned}
\sum_{\mathrm{pq}} \sum_{\mathrm{i}}^{\mathrm{occ}}(\mathrm{pq} \mid \mathrm{ii}) P_{\mathrm{pq}} & =\sum_{\mathrm{pq}} \sum_{\mathrm{i}}^{\mathrm{occ}} \sum_{\mu \nu \kappa \lambda}(\mu \nu \mid \kappa \lambda) C_{\kappa \mathrm{i}} C_{\lambda \mathrm{i}} C_{\mu \mathrm{p}} C_{\mathrm{vq}} P_{\mathrm{pq}} \\
& =\sum_{\mu \nu \kappa \lambda}(\mu \nu \mid \kappa \lambda) P_{\mu \nu} D_{\kappa \lambda},
\end{aligned}
$$

where

$$
D_{\mu v}=\sum_{\mathrm{i}}^{\mathrm{occ}} C_{\mu \mathrm{i}} C_{\mathrm{vi}}
$$

is an element of the ground-state density matrix. The third term reads

$$
\begin{gathered}
\sum_{\mathrm{pq}}\left(v_{\mathrm{pq}}^{\mathrm{XC}}+v_{\mathrm{pq}}^{\mathrm{emb}}\right) P_{\mathrm{pq}}=\sum_{\mathrm{pq}} \sum_{\mu \nu}\left(v_{\mu v}^{\mathrm{XC}}+v_{\mu v}^{\mathrm{emb}}\right) C_{\mu \mathrm{p}} C_{v \mathrm{q}} P_{\mathrm{pq}} \\
=\sum_{\mu \nu}\left(v_{\mu \nu}^{\mathrm{XC}}+v_{\mu \nu}^{\mathrm{emb}}\right) P_{\mu v} .
\end{gathered}
$$

Similarly, the first part of the fifth term can be re-written as

$$
\sum_{\mathrm{pq}, \mathrm{p} \leq \mathrm{q}} S_{\mathrm{pq}} W_{\mathrm{pq}}=\sum_{\mathrm{p} \leq \mathrm{q}} \sum_{\mu \nu} S_{\mu \nu} C_{\mu \mathrm{p}} C_{\mathrm{vq}} W_{\mathrm{pq}}=\sum_{\mu \nu} S_{\mu \nu} W_{\mu \nu},
$$

where the last equality defines $W_{\mu \nu}$. For the fourth term, involving the double sum, we get

$$
\begin{aligned}
\sum_{\mathrm{ia}, \mathrm{jb}} & (X+Y)_{\mathrm{ia}}(X+Y)_{\mathrm{jb}}\left[(\mathrm{ia} \mid \mathrm{jb})+f_{\mathrm{ia}, \mathrm{jb}}^{\mathrm{XC}}+f_{\mathrm{XC}, \mathrm{ia}, \mathrm{jb}}^{\mathrm{nadd}}+f_{\mathrm{kin}, \mathrm{ia}, \mathrm{jb}}^{\mathrm{nadd}}\right] \\
= & \sum_{\mu v \kappa \lambda} \sum_{\mathrm{ia}, \mathrm{jb}}(X+Y)_{\mathrm{ia}}(X+Y)_{\mathrm{jb}} C_{\mu \mathrm{i}} C_{\mathrm{va}} C_{\mathrm{\kappa j}} C_{\lambda \mathrm{b}} \\
& \times\left[(\mu v \mid \kappa \lambda)+f_{\mu \nu, \kappa \lambda}^{\mathrm{XC}}+f_{\mathrm{XC}, \mu \nu, \kappa \lambda}^{\mathrm{nadd}}+f_{\mathrm{kin}, \mu v, \kappa \lambda}^{\mathrm{nadd}}\right] \\
= & \sum_{\mu v \kappa \lambda}(X+Y)_{\mu \nu}(X+Y)_{\kappa \lambda} \\
& \times\left[(\mu v \mid \kappa \lambda)+f_{\mu \nu, \kappa \lambda}^{\mathrm{XC}}+f_{\mathrm{XC}, \mu \nu, \kappa \lambda}^{\mathrm{nadd}}+f_{\mathrm{kin}, \mu v, \kappa \lambda}^{\mathrm{nadd}}\right] .
\end{aligned}
$$

Grouping everything together, the Lagrangian in terms of atomic integrals is established (except for the last two terms, which will not survive the differentiation as shown in the following section),

$$
\begin{aligned}
& L=\sum_{\mu \nu} h_{\mu v} P_{\mu v}+\sum_{\mu v \kappa \lambda}(\mu v \mid \kappa \lambda)\left[P_{\mu v} D_{\kappa \lambda}+(X+Y)_{\mu v}(X+Y)_{\kappa \lambda}\right] \\
& +\sum_{\mu \nu}\left(v_{\mu \nu}^{\mathrm{XC}}+v_{\mu \nu}^{\mathrm{emb}}\right) P_{\mu v}+\sum_{\mu v \kappa \lambda}(X+Y)_{\mu \nu}(X+Y)_{\kappa \lambda} \\
& \times\left(f_{\mu \nu, \kappa \lambda}^{\mathrm{XC}}+f_{\mathrm{XC}, \mu \nu, \kappa \lambda}^{\mathrm{nadd}}+f_{\mathrm{kin}, \mu \nu, \kappa \lambda}^{\mathrm{nadd}}\right) \\
& -\sum_{\mu \nu} S_{\mu \nu} W_{\mu \nu}+\sum_{\mathrm{p}} W_{\mathrm{pp}}-\omega\left[\sum_{\mathrm{ia}}(X+Y)_{\mathrm{ia}}(X-Y)_{\mathrm{ia}}-1\right] .
\end{aligned}
$$

\section{A.5 Derivative of the Lagrangian in terms of AO integrals and densities}

At this point the dependency of the Lagrangian, $L$, on $\xi$ has been clearly established, and it is apparent that only atomicbasis-function integrals are involved in the differentiation with respect to $\xi$, i.e., $L^{\xi}=L^{(\xi)}$ by means of the conditions in eqn (103). Then, after taking the derivative with respect to $\xi$, the following result occurs $[\xi$ as a superscript indicates differentiation over $\xi$, while $(\xi)$ means that MO coefficients are kept constant during differentiation] 


$$
\begin{aligned}
L^{\xi}= & \sum_{\mu \nu} h_{\mu \nu}^{\xi} P_{\mu \nu}+\sum_{\mu \nu \kappa \lambda}(\mu \nu \mid \kappa \lambda)^{\xi}\left[P_{\mu \nu} D_{\kappa \lambda}+(X+Y)_{\mu \nu}(X+Y)_{\kappa \lambda}\right] \\
& +\sum_{\mu \nu}\left[\left(v_{\mu \nu}^{\mathrm{XC}}\right)^{(\xi)}+\left(v_{\mu \nu}^{\mathrm{emb}}\right)^{(\xi)}\right] P_{\mu \nu}-\sum_{\mu \nu} S_{\mu \nu}^{\xi} W_{\mu \nu} \\
& +\sum_{\mu \nu \kappa \lambda}(X+Y)_{\mu \nu}(X+Y)_{\kappa \lambda}\left(f_{\mu \nu, \kappa \lambda}^{\mathrm{XC}(\xi)}+f_{\mathrm{XC}, \mu v, \kappa \lambda}^{\mathrm{nadd}(\xi)}+f_{\mathrm{kin}, \mu v, \kappa \lambda}^{\mathrm{nadd}(\xi)}\right) .
\end{aligned}
$$

In the context of a DFT framework, it is more convenient to write this expression in terms of densities, in particular, for the purpose of implementation in the ADF code. Using the definition $\rho^{\mathrm{M}}=\sum_{\mathrm{mn}} \phi_{\mathrm{m}} \phi_{\mathrm{n}} M_{\mathrm{mn}}=\sum_{\mu \nu} \chi_{\mu} \chi_{v} M_{\mu v}$, eqn (114) can be rewritten in terms of densities. ${ }^{10}$ The Coulomb potential based on this density takes the following form (for ease of notation the integration of a function $\int f(\mathbf{r}) \mathrm{d} \mathbf{r}$ will be defined as $\left.\int f\right)$,

$$
v_{\mathrm{C}}^{\mathrm{M}}=\sum_{\mu v} \int \frac{1}{r_{12}} \chi_{\mu} \chi_{v} M_{\mu v}=\int \frac{1}{r_{12}} \rho^{\mathrm{M}} .
$$

Eqn (114) will be analyzed term by term. Defining the kinetic energy operator as $\hat{T}_{\mathrm{s}}=-\frac{1}{2} \nabla^{2}$ the first term from eqn (114) will take the following form:

$$
\begin{aligned}
\sum_{\mu \nu} h_{\mu \nu}^{\xi} P_{\mu v}= & \sum_{\mu \nu} \int\left[\chi_{\mu}\left(\hat{T}_{\mathrm{s}}+\hat{v}_{\mathrm{ext}}\right) \chi_{v}\right]^{\xi} P_{\mu \nu}=\int \sum_{\mu \nu}\left(\chi_{\mu} \hat{T}_{\mathrm{s}} \chi_{v}\right)^{\xi} P_{\mu \nu} \\
& +\int v_{\mathrm{ext}}^{\xi} \sum_{\mu \nu}\left(\chi_{\mu} \chi_{v}\right) P_{\mu v}+\int v_{\mathrm{ext}} \sum_{\mu \nu}\left(\chi_{\mu} \chi_{v}\right)^{\xi} P_{\mu \nu} \\
= & T_{\mathrm{s}}\left[\rho^{\mathrm{P}(\xi)}\right]+\int v_{\mathrm{ext}}^{\xi} \rho^{\mathrm{P}}+\int v_{\mathrm{ext}} \rho^{\mathrm{P}(\xi)} .
\end{aligned}
$$

The sum containing derivatives of the matrix elements of the XC potential read

$$
\sum_{\mu \nu}\left(v_{\mu v}^{\mathrm{XC}}\right)^{(\xi)} P_{\mu \nu}=\int \rho^{\mathrm{P}(\xi)} v^{\mathrm{XC}}+\int \rho^{\mathrm{P}} f^{\mathrm{XC}} \rho^{(\xi)} .
$$

In the same way one can transform the sum with the derivatives of the embedding potential matrix elements

$$
\begin{aligned}
& \sum_{\mu v}\left(v_{\mu v}^{\mathrm{emb}}\right)^{(\xi)} P_{\mu v}=\int \sum_{\mu v}\left(\chi_{\mu} \chi_{v}\right)^{\xi} P_{\mu v} v_{\mathrm{ext}}^{\mathrm{f}} \\
& +\sum_{\mu v \kappa_{\mathrm{f}} \lambda_{\mathrm{f}}}\left(\mu v \mid \kappa_{\mathrm{f}} \lambda_{\mathrm{f}}\right)^{\xi} P_{\mu v} D_{\kappa_{\mathrm{f}} \lambda_{\mathrm{f}}} \\
& +\sum_{\mu \nu}\left(v_{\mu \nu, \mathrm{kin}}^{\mathrm{nadd}}\right)^{(\xi)} P_{\mu \nu}+\sum_{\mu \nu}\left(v_{\mu \nu, \mathrm{XC}}^{\mathrm{nadd}}\right)^{(\xi)} P_{\mu \nu} \\
& =\int \rho^{\mathrm{P}(\xi)} v_{\mathrm{ext}}^{\mathrm{f}}+\int \rho^{\mathrm{P}(\xi)} v_{\mathrm{C}}^{\mathrm{f}} \\
& +\int \rho^{\mathrm{P}(\xi)} v_{\mathrm{XC}}^{\text {nadd }}+\int \rho^{\mathrm{P}(\xi)} v_{\text {kin }}^{\text {nadd }}+\int \rho^{\mathrm{P}} f_{\mathrm{XC}}^{\text {nadd }} \rho^{(\xi)} \\
& +\int \rho^{\mathrm{P}} f_{\text {kin }}^{\text {nadd }} \rho^{(\xi)} .
\end{aligned}
$$

Here, we have explicitly assumed that the external potential from the frozen region does not change, i.e., no perturbations in the positions of frozen atoms are included (although this can easily be generalized). The derivative of the term containing the kernels in eqn (114) takes the following form:

$$
\begin{gathered}
\sum_{\mu v \kappa \lambda}(X+Y)_{\mu \nu}(X+Y)_{\kappa \lambda}\left(f_{\mu v, \kappa \lambda}^{\mathrm{XC}(\xi)}+f_{\mathrm{XC}, \mu \nu, \kappa \lambda}^{\mathrm{nadd}(\xi)}+f_{\mathrm{kin}, \mu \nu, \kappa \lambda}^{\mathrm{nadd}(\xi)}\right) \\
=2 \int \rho^{(X+Y)(\xi)} f^{\mathrm{XC}} \rho^{(X+Y)}+2 \int \rho^{(X+Y)(\xi)} f_{\mathrm{XC}}^{\mathrm{nadd}} \rho^{(X+Y)} \\
\quad+2 \int \rho^{(X+Y)(\xi)} f_{\mathrm{kin}}^{\mathrm{nadd}} \rho^{(X+Y)}+\int \rho^{(X+Y)} \rho^{(X+Y)} \rho^{(\xi)} g^{\mathrm{XC}} \\
+\int \rho^{(X+Y)} \rho^{(X+Y)} \rho^{(\xi)} g_{\mathrm{XC}}^{\text {nadd }}+\int \rho^{(X+Y)} \rho^{(X+Y)} \rho^{(\xi)} g_{\mathrm{kin}}^{\text {nadd }}
\end{gathered}
$$

The term with the Coulomb integrals which originates from the Fock matrix and from the TDDFT part can be transformed as follows:

$$
\begin{gathered}
\sum_{\mu v \kappa \lambda}(\mu v \mid \kappa \lambda)^{\xi}\left[P_{\mu v} D_{\kappa \lambda}+(X+Y)_{\mu v}(X+Y)_{\kappa \lambda}\right] \\
=\int \rho^{\mathrm{P}(\xi)} v_{\mathrm{C}}+\int v_{\mathrm{C}}^{\mathrm{P}} \rho^{(\xi)}+2 \int \rho^{(X+Y)(\xi)} v_{\mathrm{C}}^{(X+Y)} .
\end{gathered}
$$

Grouping together all the terms mentioned above, it is finally possible to write the derivative of the Lagrangian in terms of densities

$$
\begin{aligned}
L^{\xi}= & T_{\mathrm{S}}\left[\rho^{\mathrm{P}(\xi)}\right] \\
& +\int \rho^{\mathrm{P}(\xi)}\left(v_{\mathrm{ext}}+v_{\mathrm{C}}+v^{\mathrm{XC}}+v_{\mathrm{ext}}^{\mathrm{f}}+v_{\mathrm{C}}^{\mathrm{f}}+v_{\mathrm{XC}}^{\mathrm{nadd}}+v_{\mathrm{kin}}^{\mathrm{nadd}}\right) \\
& +\int\left(v_{\mathrm{ext}}^{\xi} \rho^{\mathrm{P}}+v_{\mathrm{C}}^{\mathrm{P}} \rho^{(\xi)}\right)+\int \rho^{\mathrm{P}}\left(f^{\mathrm{XC}}+f_{\mathrm{XC}}^{\mathrm{nadd}}+f_{\text {kin }}^{\mathrm{nadd}}\right) \rho^{(\xi)} \\
& +2 \int \rho^{(X+Y)(\xi)}\left[v_{\mathrm{C}}^{(X+Y)}+\left(f^{\mathrm{XC}}+f_{\mathrm{XC}}^{\mathrm{nadd}}+f_{\text {kin }}^{\text {nadd }}\right) \rho^{(X+Y)}\right] \\
& +\int \rho^{(X+Y)} \rho^{(X+Y)} \rho^{(\xi)}\left[g^{\mathrm{XC}}+g_{\mathrm{XC}}^{\mathrm{nadd}}+g_{\text {kin }}^{\mathrm{nadd}}\right]-\sum_{\mu \nu} S_{\mu \nu}^{\xi} W_{\mu \nu} .
\end{aligned}
$$

\section{A.6 Derivation of the $Z$-vector equation}

For the calculation of the Lagrangian derivative, eqn (114) and (121), the elements of $\mathbf{W}$ and $\mathbf{Z}$ have to be known. The matrix $\mathbf{P}$ contains the difference between the excited- and ground-state density matrices

$$
\mathbf{P}=\mathbf{Z}+\mathbf{T},
$$

where $\mathbf{T}$ is the unrelaxed difference density matrix ("unrelaxed" part) and is already determined once the TDDFT eigenvalue problem has been solved, since it contains products of transition density matrices (excitation vectors, TDDFT 
solution factors). The matrix $\mathbf{Z}$ takes care of the relaxation of the ground-state orbitals and can be of the same order of magnitude as $\mathbf{T}^{47}$ The information in $\mathbf{P}$ is complementary to the information contained in the transition vector $|\mathbf{X}, \mathbf{Y}\rangle$ (the transition density matrix). ${ }^{47} \mathbf{P}$ is related to the difference of expectation values for excited states and the ground state, while the transition vector is related to mixed matrix elements between the ground and excited states. $\mathbf{P}$ can give insight into the re-distribution of the electronic charge due to the excitation process. $^{47}$

These Lagrangian multipliers can be obtained from the so-called $Z$-vector equation, ${ }^{5,37}$ which can be derived from the following condition:

$$
\frac{\partial L}{\partial C_{\mu \mathrm{p}}}=0 .
$$

If eqn (123) is multiplied by $C_{\mu \mathrm{q}}$ and summed over index $\mu$, it leads to the following equation:

$$
Q_{\mathrm{pq}}+\sum_{\text {ia }} Z_{\mathrm{ia}} \sum_{\mu} \frac{\partial F_{\mathrm{ia}}}{\partial C_{\mu \mathrm{p}}} C_{\mu \mathrm{q}}=\sum_{\mathrm{rs}, \mathrm{r} \leq \mathrm{s}} W_{\mathrm{rs}} \sum_{\mu} \frac{\partial S_{\mathrm{rs}}}{\partial C_{\mu \mathrm{p}}} C_{\mu \mathrm{q}},
$$

where $\mathbf{Q}$ is a matrix with elements,

$$
Q_{\mathrm{pq}}=\sum_{\mu} \frac{\partial G[X, Y, \omega]}{\partial C_{\mu \mathrm{p}}} C_{\mu \mathrm{q}} .
$$

Four possible cases for the orbitals p and $q$ in eqn (124) have to be considered at this point. The first case corresponds to occupied orbitals for $\mathrm{p}$ and $\mathrm{q}$, so that we can set $\mathrm{p}=\mathrm{k} \leq \mathrm{q}=\mathrm{l}$. The right hand-side of eqn (124) can be expanded as follows:

$$
\begin{aligned}
& \sum_{\mathrm{rs}, \mathrm{r} \leq \mathrm{s}} W_{\mathrm{rs}} \sum_{\mu} \frac{\partial S_{\mathrm{rs}}}{\partial C_{\mu \mathrm{k}}} C_{\mu \mathrm{l}} \\
& =\sum_{\mathrm{rs}, \mathrm{r} \leq \mathrm{s}} W_{\mathrm{rs}} \sum_{\mu} \frac{\partial\left(\sum_{\lambda \rho} C_{\lambda \mathrm{r}} C_{\rho \mathrm{s}} S_{\lambda \rho}\right)}{\partial C_{\mu \mathrm{k}}} C_{\mu \mathrm{l}} \\
& =\sum_{\mathrm{rs}, \mathrm{r} \leq \mathrm{s}} W_{\mathrm{rs}}\left(\sum_{\mu \rho} C_{\mu \mathrm{l}} C_{\rho \mathrm{s}} S_{\mu \rho} \delta_{\mathrm{rk}}+\sum_{\lambda \mu} C_{\lambda \mathrm{r}} C_{\mu \mathrm{l}} S_{\lambda \mu} \delta_{\mathrm{ks}}\right) \\
& =\sum_{\mathrm{rs}, \mathrm{r} \leq \mathrm{s}} W_{\mathrm{rs}}\left(\delta_{\mathrm{rk}} S_{\mathrm{ls}}+\delta_{\mathrm{ks}} S_{\mathrm{rl}}\right)=\sum_{\mathrm{s}, \mathrm{s} \geq \mathrm{k}} W_{\mathrm{ks}} S_{\mathrm{ls}}+\sum_{\mathrm{r}, \mathrm{r} \leq \mathrm{k}} W_{\mathrm{rk}} S_{\mathrm{rl}} .
\end{aligned}
$$

Taking into account that $W_{\mathrm{rs}}=W_{\mathrm{sr}}$, the following equation holds for any $\mathrm{k}$,

$$
\sum_{\mathrm{t}} W_{\mathrm{kt}} S_{\mathrm{lt}}+W_{\mathrm{kk}} S_{\mathrm{kl}}=W_{\mathrm{kl}}\left(1+\delta_{\mathrm{kl}}\right) .
$$

The last equality holds because of $S_{\mathrm{tk}}=\delta_{\mathrm{tk}}$. The second term of the left hand-side of eqn (124) can be transformed by analogy with the derivation mentioned above

$$
\begin{aligned}
& \sum_{\text {ia }} Z_{\text {ia }} \sum_{\mu} \frac{\partial F_{\mathrm{ia}}}{\partial C_{\mu \mathrm{k}}} C_{\mu \mathrm{l}} \\
& =2 \sum_{\mathrm{ia}} Z_{\mathrm{ia}} \underbrace{\left[h_{\mathrm{la}}+\sum_{\mathrm{m}}(\mathrm{la} \mid \mathrm{mm})+v_{\mathrm{la}}^{\mathrm{XC}}+v_{\mathrm{la}}^{\mathrm{emb}}\right]}_{F_{\mathrm{la}}} \delta_{\mathrm{ki}} \\
& +2 \sum_{\mathrm{ia}} Z_{\mathrm{ia}} \underbrace{\left[h_{\mathrm{il}}+\sum_{\mathrm{m}}(\mathrm{il} \mid \mathrm{mm})+v_{\mathrm{il}}^{\mathrm{XC}}+v_{\mathrm{il}}^{\mathrm{emb}}\right]}_{F_{\mathrm{il}}} \delta_{\mathrm{ka}} \\
& +\underbrace{\sum_{\mathrm{ia}} 2 Z_{\mathrm{ia}}\left[(\mathrm{ia} \mid \mathrm{kl})+f_{\mathrm{ia}, \mathrm{kl}}^{\mathrm{XC}}+f_{\mathrm{XC}, \mathrm{ia}, \mathrm{kl}}^{\mathrm{nadd}}+f_{\mathrm{kin}, \mathrm{ia}, \mathrm{kl}]}^{\mathrm{nadd}}\right]}_{H_{\mathrm{ia}}+[\mathbf{Z}]}
\end{aligned}
$$

The first sum on the right hand-side of eqn (128) is zero due to the condition $F_{\mathrm{la}}=0$. Since $\delta_{\mathrm{ka}}=0$ for every a, the second term of eqn (128) also vanishes, and finally eqn (124) changes to

$$
Q_{\mathrm{kl}}+H_{\mathrm{kl}}^{+}[\mathbf{Z}]=W_{\mathrm{kl}}\left(1+\delta_{\mathrm{kl}}\right) \text {. }
$$

For the second case, where only virtual orbitals are considered for $\mathrm{p}$ and $\mathrm{q}, \mathrm{p}=\mathrm{c} \leq \mathrm{q}=\mathrm{d}$ in eqn (124), the result for the terms containing $W_{\mathrm{rs}}$ will be similar to the previous case, namely $W_{\text {cd }}\left(1+\delta_{\text {cd }}\right)$. The second term of the left-hand side in eqn (124) transforms as follows in this case:

$$
\begin{gathered}
\sum_{\text {ia }} Z_{\mathrm{ia}} \sum_{\mu} \frac{\partial F_{\mathrm{ia}}}{\partial C_{\mu \mathrm{c}}} C_{\mu \mathrm{d}}=2 \sum_{\mathrm{ia}} Z_{\mathrm{ia}} \underbrace{\left[h_{\mathrm{da}}+\sum_{\mathrm{m}}(\mathrm{da} \mid \mathrm{mm})+v_{\mathrm{da}}^{\mathrm{XC}}+v_{\mathrm{da}}^{\mathrm{emb}}\right]}_{F_{\mathrm{da}}} \delta_{\mathrm{ci}} \\
+2 \sum_{\mathrm{ia}} Z_{\mathrm{ia}} \underbrace{\left[h_{\mathrm{id}}+\sum_{\mathrm{m}}(\mathrm{id} \mid \mathrm{mm})+v_{\mathrm{id}}^{\mathrm{XC}}+v_{\mathrm{id}}^{\mathrm{emb}}\right]}_{F_{\mathrm{id}}} \delta_{\mathrm{ca}}=0 .
\end{gathered}
$$

Since $\delta_{\mathrm{ci}}=0$ for every $\mathrm{i}$, the first term on the right-hand side of eqn (130) vanishes and the second term is zero due to the condition $F_{\text {id }}=0$. Then eqn (124) can be simplified for the case $\mathrm{p}=\mathrm{c} \leq \mathrm{q}=\mathrm{d}$ to

$$
Q_{\mathrm{cd}}=W_{\mathrm{cd}}\left(1+\delta_{\mathrm{cd}}\right) .
$$

The third case concerns the combination of virtual (c) and occupied (k) orbitals, so that $\mathrm{p}=\mathrm{k}, \mathrm{q}=\mathrm{c}$ in eqn (124),

$$
\begin{aligned}
& \sum_{\mathrm{rs}, \mathrm{r} \leq \mathrm{s}} W_{\mathrm{rs}} \sum_{\mu} \frac{\partial S_{\mathrm{rs}}}{\partial C_{\mu \mathrm{k}}} C_{\mu \mathrm{c}} \\
& =\sum_{\mathrm{rs}, \mathrm{r} \leq \mathrm{s}} W_{\mathrm{rs}} \sum_{\mu} \frac{\partial\left(\sum_{\lambda \rho} C_{\lambda \mathrm{r}} C_{\rho \mathrm{s}} S_{\lambda \rho}\right)}{\partial C_{\mu \mathrm{k}}} C_{\mu \mathrm{c}} \\
& =\sum_{\mathrm{rs}, \mathrm{r} \leq \mathrm{s}} W_{\mathrm{rs}}\left(\sum_{\mu, \rho} C_{\mu, \mathrm{c}} C_{\rho, \mathrm{s}} S_{\mu \rho} \delta_{\mathrm{rk}}+\sum_{\lambda, \mu} C_{\lambda, \mathrm{r}} C_{\mu, \mathrm{c}} S_{\lambda \mu} \delta_{\mathrm{ks}}\right) \\
& =\sum_{\mathrm{rs}, \mathrm{r} \leq \mathrm{s}} W_{\mathrm{rs}}\left(\delta_{\mathrm{rk}} S_{\mathrm{cs}}+\delta_{\mathrm{ks}} S_{\mathrm{rc}}\right)=\sum_{\mathrm{s}, \mathrm{s} \geq \mathrm{k}} W_{\mathrm{ks}} S_{\mathrm{cs}}+\sum_{\mathrm{r}, \mathrm{r} \leq \mathrm{k}} W_{\mathrm{rk}} S_{\mathrm{rc}}
\end{aligned}
$$


Taking into account that $W_{\mathrm{rs}}=W_{\mathrm{sr}}$, the following equation holds for a given $\mathrm{k}$

$$
\sum_{\mathrm{s}, \mathrm{s} \geq \mathrm{k}} W_{\mathrm{ks}} S_{\mathrm{cs}}+\sum_{\mathrm{r}, \mathrm{r} \leq \mathrm{k}} W_{\mathrm{rk}} S_{\mathrm{rc}}=\sum_{\mathrm{t}} W_{\mathrm{tk}} S_{\mathrm{tc}}+W_{\mathrm{kk}} S_{\mathrm{kc}}=W_{\mathrm{kc}} .
$$

The last equality holds true since $S_{\mathrm{tc}}=\delta_{\mathrm{tc}}$ and $\mathrm{k} \neq \mathrm{c}$. The second term on the left-hand side of eqn (124) can then be rewritten in the following way:

$$
\begin{aligned}
\sum_{\text {ia }} Z_{\text {ia }} \sum_{\mu} \frac{\partial F_{\mathrm{ia}}}{\partial C_{\mu \mathrm{k}}} C_{\mu \mathrm{c}} \\
=2 \sum_{\mathrm{ia}} Z_{\mathrm{ia}} \underbrace{\left[h_{\mathrm{ca}}+\sum_{\mathrm{m}}(\mathrm{ca} \mid \mathrm{mm})+v_{\mathrm{ca}}^{\mathrm{XC}}+v_{\mathrm{ca}}^{\mathrm{emb}}\right]}_{F_{\mathrm{ca}}} \delta_{\mathrm{ik}} \\
+2 \sum_{\mathrm{ia}} Z_{\mathrm{ia}} \underbrace{\left[h_{\mathrm{ic}}+\sum_{\mathrm{m}}(\mathrm{ic} \mid \mathrm{mm})+v_{\mathrm{ic}}^{\mathrm{XC}}+v_{\mathrm{ic}}^{\mathrm{emb}}\right]}_{F_{\mathrm{ic}}} \delta_{\mathrm{ka}} \\
+2 \underbrace{}_{H_{\mathrm{ia}} Z_{\mathrm{ia}}\left[(\mathrm{ia} \mid \mathrm{kc})+f_{\mathrm{ia}, \mathrm{kc}}^{\mathrm{XC}}+f_{\mathrm{XC}, \mathrm{ia}, \mathrm{kc}}^{\mathrm{nadd}}+f_{\mathrm{kin}, \mathrm{ia}, \mathrm{kc}}^{\mathrm{nadd}}\right]}
\end{aligned}
$$

Since $\delta_{\mathrm{ka}}=0$ for every a, the second term vanishes. In terms of canonical orbitals, the equation above changes to,

$$
\sum_{\mathrm{ia}} Z_{\mathrm{ia}} \sum_{\mu} \frac{\partial F_{\mathrm{ia}}}{\partial C_{\mu \mathrm{k}}} C_{\mu \mathrm{c}}=\varepsilon_{\mathrm{c}} Z_{\mathrm{kc}}+H_{\mathrm{kc}}^{+}[\mathbf{Z}]
$$

Eqn (124) will change to

$$
Q_{\mathrm{kc}}+\varepsilon_{\mathrm{c}} Z_{\mathrm{kc}}+H_{\mathrm{kc}}{ }^{+}[\mathbf{Z}]=W_{\mathrm{kc}} .
$$

Finally, the fourth case concerns the combination of occupied and virtual orbitals by making the choice $\mathrm{p}=\mathrm{c}, \mathrm{q}=\mathrm{k}$. The righthand side of eqn (124) will give the same result as in the case above $\left(W_{\mathrm{kc}}\right)$, while the second term on the left hand side will take the form

$$
\begin{aligned}
& \sum_{\mathrm{ia}} Z_{\mathrm{ia}} \sum_{\mu} \frac{\partial F_{\mathrm{ia}}}{\partial C_{\mu \mathrm{c}}} C_{\mu \mathrm{k}} \\
& =2 \sum_{\mathrm{ia}} Z_{\mathrm{ia}} \underbrace{\left[h_{\mathrm{ka}}+\sum_{\mathrm{m}}(\mathrm{ka} \mid \mathrm{mm})+v_{\mathrm{ka}}^{\mathrm{XC}}+v_{\mathrm{ka}}^{\mathrm{emb}}\right]}_{F_{\mathrm{ka}}} \delta_{\mathrm{ic}} \\
& +2 \sum_{\mathrm{ia}} Z_{\mathrm{ia}} \underbrace{\left[h_{\mathrm{ik}}+\sum_{\mathrm{m}}(\mathrm{ik} \mid \mathrm{mm})+v_{\mathrm{ik}}^{\mathrm{XC}}+v_{\mathrm{ik}}^{\mathrm{emb}}\right]}_{F_{\mathrm{ik}}} \delta_{\mathrm{ac}}=\varepsilon_{\mathrm{k}} Z_{\mathrm{kc}} .
\end{aligned}
$$

The last step holds after making use of canonical orbitals and realizing that $\delta_{\mathrm{ic}}=0$ for every $\mathrm{i}$ (the first term vanishes). Finally eqn (124) takes the form

$$
Q_{\mathrm{ck}}+\varepsilon_{\mathrm{k}} Z_{\mathrm{kc}}=W_{\mathrm{kc}}
$$

Thus, the following set of equations has to be solved in order to get the vectors $\mathbf{Z}$ and $\mathbf{W}$,

$$
\begin{gathered}
Q_{\mathrm{kl}}+H_{\mathrm{kl}}^{+}[\mathbf{Z}]=W_{\mathrm{kl}}\left(1+\delta_{\mathrm{kl}}\right), \\
Q_{\mathrm{kc}}+\varepsilon_{\mathrm{c}} Z_{\mathrm{kc}}+H_{\mathrm{kc}}^{+}[\mathbf{Z}]=W_{\mathrm{kc}}, \\
Q_{\mathrm{ck}}+\varepsilon_{\mathrm{k}} Z_{\mathrm{kc}}=W_{\mathrm{kc}}, \\
Q_{\mathrm{cd}}=W_{\mathrm{cd}}\left(1+\delta_{\mathrm{cd}}\right) .
\end{gathered}
$$

Subtracting eqn (141) from eqn (140), we get

$$
\left(\varepsilon_{\mathrm{a}}-\varepsilon_{\mathrm{i}}\right) Z_{\mathrm{ia}}+H_{\mathrm{ia}}^{+}[\mathrm{Z}]=R_{\mathrm{ia}},
$$

where $R_{\mathrm{ia}}=-\left(Q_{\mathrm{ia}}-Q_{\mathrm{ai}}\right)$. It is common to call eqn (143) the $Z$-vector equation in the context of TDDFT, and it is usually written in the following form

$$
\sum_{\mathrm{jb}}(A+B)_{\mathrm{ia}, \mathrm{jb}} Z_{\mathrm{jb}}=R_{\mathrm{ia}}
$$

Once this equation is solved with respect to $\mathbf{Z}$, it is possible to determine $\mathbf{W}$ from the following equations:

$$
\begin{gathered}
W_{\mathrm{ij}}=\frac{\left(Q_{\mathrm{ij}}+H_{\mathrm{ij}}^{+}[\mathbf{Z}]\right)}{\left(1+\delta_{\mathrm{ij}}\right)}, \\
W_{\mathrm{ab}}=\frac{Q_{\mathrm{ab}}}{\left(1+\delta_{\mathrm{ab}}\right)},
\end{gathered}
$$

$$
W_{\mathrm{ia}}=Q_{\mathrm{ai}}+\varepsilon_{\mathrm{i}} Z_{\mathrm{ia}} .
$$

To solve the $Z$-vector equation, eqn (144), explicit expressions for the elements of the vector $\mathbf{Q}$ have to be established:

$$
Q_{\mathrm{pq}}=\sum_{\mu} \frac{\partial G[X, Y, \omega]}{\partial C_{\mu \mathrm{p}}} C_{\mu \mathrm{q}} .
$$

These expressions are derived in Section B.

\section{B Elements of the vector $Q$}

Here we present a derivation of the expressions for elements of the vector Q. First, two auxiliary relations will be derived in Section B.1 and then the necessary expressions will be obtained in Section B.2.

\section{B.1 Auxiliary relation for the derivation of elements $Q_{\mathrm{pq}}$}

If differentiation of eqn (78) with respect to $(X+Y)_{\text {la }}$ [note that $(X+Y)_{\mathrm{ia}}$ and $(X-Y)_{\mathrm{ia}}$ are considered as independent variables] is followed by multiplication with $(X+Y)_{\mathrm{ka}}$ and summation over a, then due to the condition in eqn (79), the following equation is valid: 


$$
\begin{aligned}
& \sum_{\mathrm{a}} \frac{\partial}{\partial(X+Y)_{\mathrm{la}}} \frac{1}{2}\left[\sum_{\mathrm{ia}, \mathrm{jb}}(X-Y)_{\mathrm{ia}}(A-B)_{\mathrm{ia}, \mathrm{jb}}(X-Y)_{\mathrm{jb}}\right. \\
& \left.\quad+\sum_{\mathrm{ia}, \mathrm{jb}}(X+Y)_{\mathrm{ia}}(A+B)_{\mathrm{ia}, \mathrm{jb}}(X+Y)_{\mathrm{jb}}\right](X+Y)_{\mathrm{ka}} \\
& \quad=\sum_{\mathrm{a}} \frac{\partial}{\partial(X+Y)_{\mathrm{la}}}\left[\omega \sum_{\mathrm{ia}}(X-Y)_{\mathrm{ia}}(X+Y)_{\mathrm{ia}}-1\right](X+Y)_{\mathrm{ka}} .
\end{aligned}
$$

After some manipulation, the previous equation can be transformed to

$$
\sum_{\mathrm{a}, \mathrm{jb}}(X+Y)_{\mathrm{ka}}(A+B)_{\mathrm{la}, \mathrm{jb}}(X+Y)_{\mathrm{jb}}=\omega \sum_{\mathrm{a}}(X-Y)_{\mathrm{la}}(X+Y)_{\mathrm{ka}} .
$$

Using eqn (13) and (14), this can be rewritten as

$$
\begin{aligned}
& \sum_{\mathrm{ab}}(X+Y)_{\mathrm{ka}}(X+Y)_{\mathrm{lb}} F_{\mathrm{ab}}-\sum_{\mathrm{ja}}(X+Y)_{\mathrm{ka}}(X+Y)_{\mathrm{ja}} F_{\mathrm{lj}} \\
& +\sum_{\mathrm{a}, \mathrm{jb}}(X+Y)_{\mathrm{ka}} \times 2\left[(\mathrm{la} \mid \mathrm{jb})+f_{\mathrm{la}, \mathrm{jb}}^{\mathrm{XC}}+f_{\mathrm{XC}, \mathrm{la}, \mathrm{jb}}^{\mathrm{nadd}}+f_{\mathrm{kin}, \mathrm{l}, \mathrm{jb}}^{\mathrm{nadd}}\right] \\
& \times(X+Y)_{\mathrm{jb}}=\omega \sum_{\mathrm{a}}(X-Y)_{\mathrm{la}}(X+Y)_{\mathrm{ka}} \cdot
\end{aligned}
$$

The differentiation of eqn (78) can also be carried out with respect to $(X-Y)_{\mathrm{la}}$. If the result is multiplied by $(X-Y)_{\mathrm{ka}}$ and summed over a, the following equation can be established using eqn (80),

$$
\begin{aligned}
& \sum_{\mathrm{a}} \frac{\partial}{\partial(X-Y)_{\mathrm{la}}} \frac{1}{2}\left[\sum_{\mathrm{ia}, \mathrm{jb}}(X-Y)_{\mathrm{ia}}(A-B)_{\mathrm{ia}, \mathrm{jb}}(X-Y)_{\mathrm{jb}}\right. \\
& \left.\quad+\sum_{\mathrm{ia}, \mathrm{jb}}(X+Y)_{\mathrm{ia}}(A+B)_{\mathrm{ia}, \mathrm{jb}}(X+Y)_{\mathrm{jb}}\right](X-Y)_{\mathrm{ka}} \\
& \quad=\sum_{\mathrm{a}} \frac{\partial}{\partial(X-Y)_{\mathrm{la}}}\left[\omega \sum_{\mathrm{ia}}(X-Y)_{\mathrm{ia}}(X+Y)_{\mathrm{ia}}-1\right](X-Y)_{\mathrm{ka}} .
\end{aligned}
$$

After some further manipulations, this can be transformed to

$$
\sum_{\mathrm{a}, \mathrm{jb}}(X-Y)_{\mathrm{ka}}(A-B)_{\mathrm{la}, \mathrm{jb}}(X-Y)_{\mathrm{jb}}=\omega \sum_{\mathrm{a}}(X-Y)_{\mathrm{ka}}(X+Y)_{\mathrm{la}} \text {. }
$$

Using eqn (13) and (14), this equation can further be rewritten as

$$
\begin{aligned}
& \sum_{\mathrm{ab}}(X-Y)_{\mathrm{ka}}(X-Y)_{\mathrm{lb}} F_{\mathrm{ab}}-\sum_{\mathrm{ja}}(X-Y)_{\mathrm{ka}}(X-Y)_{\mathrm{ja}} F_{\mathrm{lj}} \\
& \quad=\omega \sum_{\mathrm{a}}(X-Y)_{\mathrm{ka}}(X+Y)_{\mathrm{la}} .
\end{aligned}
$$

If now eqn (151) and (154) are summed up, it is possible to derive the equality,

$$
\begin{aligned}
& \sum_{\mathrm{ab}}\left[(X-Y)_{\mathrm{ka}}(X-Y)_{\mathrm{lb}}+(X+Y)_{\mathrm{ka}}(X+Y)_{\mathrm{lb}}\right] F_{\mathrm{ab}} \\
& -\sum_{\mathrm{ja}}\left[(X-Y)_{\mathrm{ka}}(X-Y)_{\mathrm{ja}}+(X+Y)_{\mathrm{ka}}(X+Y)_{\mathrm{ja}}\right] F_{\mathrm{lj}} \\
& +\sum_{\mathrm{a}, \mathrm{jb}}(X+Y)_{\mathrm{ka}} 2\left[(\mathrm{la} \mid \mathrm{jb})+f_{\mathrm{la}, \mathrm{jb}}^{\mathrm{XC}}+f_{\mathrm{XC}, \mathrm{la}, \mathrm{jb}}^{\mathrm{nadd}}+f_{\mathrm{kin}, \mathrm{la}, \mathrm{jb}}^{\mathrm{nadd}}\right](X+Y)_{\mathrm{jb}} \\
& \quad=\omega \sum_{\mathrm{a}}\left\{(X-Y)_{\mathrm{ka}}(X+Y)_{\mathrm{la}}+(X-Y)_{\mathrm{la}}(X+Y)_{\mathrm{ka}}\right\} .
\end{aligned}
$$

Representing the Fock matrix in canonical orbitals and substituting the diagonal elements by their corresponding orbital energies, the equation above changes to

$$
\begin{aligned}
& \sum_{\mathrm{a}}\left(\varepsilon_{\mathrm{a}}-\varepsilon_{\mathrm{l}}\right)\left\{(X-Y)_{\mathrm{ka}}(X-Y)_{\mathrm{la}}+(X+Y)_{\mathrm{ka}}(X+Y)_{\mathrm{la}}\right\} \\
& +\sum_{\mathrm{a}, \mathrm{jb}}(X+Y)_{\mathrm{ka}} 2\left[(\mathrm{la} \mid \mathrm{jb})+f_{\mathrm{la}, \mathrm{jb}}^{\mathrm{XC}}+f_{\mathrm{XC}, \mathrm{la}, \mathrm{jb}}^{\mathrm{nadd}}+f_{\mathrm{kin}, \mathrm{la}, \mathrm{jb}}^{\mathrm{nadd}}\right](X+Y)_{\mathrm{jb}} \\
& =\omega \sum_{\mathrm{a}}\left\{(X-Y)_{\mathrm{ka}}(X+Y)_{\mathrm{la}}+(X-Y)_{\mathrm{la}}(X+Y)_{\mathrm{ka}}\right\} .
\end{aligned}
$$

With the same strategy as before, but only differentiating eqn (78) first with respect to $(X+Y)_{\text {ic }}$ and then with respect to $(X-Y)_{\mathrm{ic}}$, each time multiplying the equation with the corresponding $(X+Y)_{\text {id }}$ or $(X-Y)_{\text {id }}$ and summing over i, it is possible to get a second important relation:

$$
\begin{gathered}
\sum_{\mathrm{i}}\left(\varepsilon_{\mathrm{d}}-\varepsilon_{\mathrm{i}}\right)\left\{(X-Y)_{\mathrm{ic}}(X-Y)_{\mathrm{id}}+(X+Y)_{\mathrm{ic}}(X+Y)_{\mathrm{id}}\right\} \\
+\sum_{\mathrm{i}, \mathrm{jb}}(X+Y)_{\mathrm{id}}(X+Y)_{\mathrm{jb}} 2\left[(\mathrm{ic} \mid \mathrm{jb})+f_{\mathrm{ic}, \mathrm{jb}}^{\mathrm{XC}}+f_{\mathrm{XC}, \mathrm{ic}, \mathrm{jb}}^{\mathrm{nadd}}+f_{\mathrm{kin}, \mathrm{ic}, \mathrm{jb}}^{\mathrm{nadd}}\right] \\
=\sum_{\mathrm{i}} \omega\left\{(X+Y)_{\mathrm{ic}}(X-Y)_{\mathrm{id}}+(X-Y)_{\mathrm{ic}}(X+Y)_{\mathrm{id}}\right\}
\end{gathered}
$$

\section{B.2 Derivation of elements $\boldsymbol{Q}_{\mathrm{pq}}$}

In order to evaluate the matrix elements $Q_{\mathrm{pq}}$ in Section A.6, eqn (148), we repeat the equation for $G[\mathbf{X}, \mathbf{Y}, \omega]$, eqn (98), at this point,

$$
\begin{aligned}
G[\mathbf{X}, \mathbf{Y}, \omega]= & \sum_{\mathrm{rs}} T_{\mathrm{rs}} F_{\mathrm{rs}}+\sum_{\mathrm{ia}, \mathrm{jb}}(X+Y)_{\mathrm{ia}}(X+Y)_{\mathrm{jb}} \\
& \times\left[(\mathrm{ia} \mid \mathrm{jb})+f_{\mathrm{ia}, \mathrm{jb}}^{\mathrm{XC}}+f_{\mathrm{XC}, \mathrm{ia}, \mathrm{jb}}^{\text {nadd }}+f_{\mathrm{kin}, \mathrm{ia}, \mathrm{jb}}^{\text {nadd }}\right] \\
& -\omega \sum_{\mathrm{ia}}\left[(X+Y)_{\mathrm{ia}}(X-Y)_{\mathrm{ia}}-1\right] .
\end{aligned}
$$

Recalling the fact that only $\left[(\mathrm{ia} \mid \mathrm{jb})+f_{\mathrm{ia}, \mathrm{jb}}^{\mathrm{XC}}+f_{\mathrm{XC}, \mathrm{ia}, \mathrm{jb}}^{\text {nadd }}+f_{\mathrm{kin}, \mathrm{ia}, \mathrm{jb}}^{\mathrm{nadd}}\right]$ and $F_{\text {rs }}$ will be affected by differentiation [see eqn (83)], again (as in Section A.6) four cases must be considered. The first case considers only occupied orbitals $\mathrm{p}$ and $\mathrm{q}$ for $Q_{\mathrm{pq}}$, so that $\mathrm{p}=$ $\mathrm{k} \leq \mathrm{q}=1$. Recalling that the terms like $v^{\mathrm{XC}}[\rho](\mathbf{r})$ and $f^{\mathrm{XC}}[\rho](\mathbf{r})$ 
implicitly depend on $C_{\mu \mathrm{k}}$ through the density $\rho$, it is possible to write

$$
\begin{aligned}
Q_{\mathrm{kl}}= & \sum_{\mu} \frac{\partial G[\mathbf{X}, \mathbf{Y}, \omega]}{\partial C_{\mu \mathrm{k}}} C_{\mu \mathrm{l}}=2 \sum_{\mathrm{rs}} T_{\mathrm{rs}} F_{\mathrm{ls}} \delta_{\mathrm{rk}} \\
& +2 \sum_{\mathrm{rs}} T_{\mathrm{rs}}\left[(\mathrm{rs} \mid \mathrm{kl})+f_{\mathrm{rs}, \mathrm{kl}}^{\mathrm{XC}}+f_{\mathrm{XC}, \mathrm{rs}, \mathrm{kl}}^{\mathrm{nadd}}+f_{\mathrm{kin}, \mathrm{rs}, \mathrm{kl}}^{\mathrm{nadd}}\right] \\
& +\sum_{\mathrm{ia}, \mathrm{jb}}(X+Y)_{\mathrm{ia}}(X+Y)_{\mathrm{jb}} \\
& \times 2\left[(\mathrm{la} \mid \mathrm{jb})+f_{\mathrm{la}, \mathrm{jb}}^{\mathrm{XC}}+f_{\mathrm{XC}, \mathrm{la}, \mathrm{jb}}^{\mathrm{nadd}}+f_{\mathrm{kin}, \mathrm{la}, \mathrm{jb}}^{\mathrm{nadd}}\right] \delta_{\mathrm{ik}} \\
& +2 \sum_{\mathrm{ia}, \mathrm{jb}}\left[g_{\mathrm{ia}, \mathrm{jb}, \mathrm{kl}}^{\mathrm{XC}}+g_{\mathrm{XC}, \mathrm{ia}, \mathrm{jb}, \mathrm{kl}}^{\mathrm{nadd}}+g_{\mathrm{kin}, \mathrm{ia}, \mathrm{jb}, \mathrm{kl}]}^{\mathrm{nadd}}\right] \\
& \times(X+Y)_{\mathrm{ia}}(X+Y)_{\mathrm{jb}} .
\end{aligned}
$$

Using the nature of the matrices $\mathbf{T}$ and $\mathbf{F}\left(T_{\mathrm{ia}}=0, F_{\mathrm{ia}}=0\right)$, and the definition of $\mathrm{H}_{\mathrm{kl}}{ }^{+}[\mathbf{M}]$, the elements $Q_{\mathrm{kl}}$ can be rewritten as

$$
\begin{aligned}
Q_{\mathrm{kl}}= & 2 \sum_{\mathrm{s}} T_{\mathrm{ks}} F_{\mathrm{ls}}+H_{\mathrm{kl}}{ }^{+}[\mathbf{T}]+\sum_{\mathrm{a}, \mathrm{jb}}(X+Y)_{\mathrm{ka}}(X+Y)_{\mathrm{jb}} \\
& \times 2\left[(\mathrm{la} \mid \mathrm{jb})+f_{\mathrm{la}, \mathrm{jb}}^{\mathrm{XC}}+f_{\mathrm{XC}, \mathrm{la}, \mathrm{jb}}^{\mathrm{nadd}}+f_{\mathrm{kin}, \mathrm{la}, \mathrm{jb}}^{\mathrm{nadd}}\right] \\
& +2 \sum_{\mathrm{ia}, \mathrm{jb}}\left[g_{\mathrm{ia}, \mathrm{jb}, \mathrm{kl}}^{\mathrm{XC}}+g_{\mathrm{XC}, \mathrm{ia}, \mathrm{jb}, \mathrm{kl}}^{\text {nadd }}+g_{\mathrm{kin}, \mathrm{ia}, \mathrm{jb}, \mathrm{kl}}^{\mathrm{nadd}}\right] \\
& \times(X+Y)_{\mathrm{ia}}(X+Y)_{\mathrm{jb}} .
\end{aligned}
$$

(160)

If one uses canonical orbitals and expands $T_{\mathrm{kl}}$ according to eqn (94), then $Q_{\mathrm{kl}}$ reads

$$
\begin{aligned}
Q_{\mathrm{kl}}= & -\varepsilon_{1} \sum_{\mathrm{a}}\left\{(X+Y)_{\mathrm{ka}}(X+Y)_{\mathrm{la}}+(X-Y)_{\mathrm{ka}}(X-Y)_{\mathrm{la}}\right\} \\
& +\sum_{\mathrm{a}, \mathrm{jb}}(X+Y)_{\mathrm{ka}}(X+Y)_{\mathrm{jb}} \\
& \times 2\left[(\mathrm{la} \mid \mathrm{jb})+f_{\mathrm{la}, \mathrm{jb}}^{\mathrm{XC}}+f_{\mathrm{XC}, \mathrm{la}, \mathrm{jb}}^{\mathrm{nadd}}+f_{\mathrm{kin}, \mathrm{la}, \mathrm{jb}}^{\mathrm{nadd}}\right]+H_{\mathrm{kl}}+[\mathbf{T}] \\
& +2 \sum_{\mathrm{ia}, \mathrm{jb}}\left[g_{\mathrm{ia}, \mathrm{jb}, \mathrm{kl}}^{\mathrm{XC}}+g_{\mathrm{XC}, \mathrm{ia}, \mathrm{jb}, \mathrm{kl}}^{\mathrm{nadd}}+g_{\mathrm{kin}, \mathrm{ia}, \mathrm{jb}, \mathrm{kl}}^{\mathrm{nadd}}\right](X+Y)_{\mathrm{ia}}(X+Y)_{\mathrm{jb}} .
\end{aligned}
$$

Now one can notice that if $\sum_{\mathrm{a}} \varepsilon_{\mathrm{a}}\left\{(X+Y)_{\mathrm{ka}}(X+Y)_{\mathrm{la}}+(X-Y)_{\mathrm{ka}}(X-Y)_{\mathrm{la}}\right\}$ is added and subtracted from the expression for element $Q_{\mathrm{kl}}$,

$$
\begin{aligned}
Q_{\mathrm{kl}}= & \sum_{\mathrm{a}}\left(\varepsilon_{\mathrm{a}}-\varepsilon_{\mathrm{l}}\right)\left\{(\mathbf{X}+\mathbf{Y})_{\mathrm{ka}}(X+Y)_{\mathrm{la}}+(X-Y)_{\mathrm{ka}}(X-Y)_{\mathrm{la}}\right\} \\
& +\sum_{\mathrm{a}, \mathrm{jb}}(X+Y)_{\mathrm{ka}}(X+Y)_{\mathrm{jb}} \\
& \times 2\left[(\mathrm{la} \mid \mathrm{jb})+f_{\mathrm{la}, \mathrm{jb}}^{\mathrm{XC}}+f_{\mathrm{XC}, \mathrm{la}, \mathrm{jb}}^{\text {nadd }}+f_{\mathrm{kin}, \mathrm{la}, \mathrm{jb}}^{\mathrm{nadd}}\right] \\
& -\sum_{\mathrm{a}} \varepsilon_{\mathrm{a}}\left\{(X+Y)_{\mathrm{ka}}(X+Y)_{\mathrm{la}}+(X-Y)_{\mathrm{ka}}(X-Y)_{\mathrm{la}}\right\}
\end{aligned}
$$

$$
\begin{aligned}
& +H_{\mathrm{kl}}{ }^{+}[\mathbf{T}]+2 \sum_{\mathrm{ia}, \mathrm{jb}}\left[g_{\mathrm{ia}, \mathrm{jb}, \mathrm{kl}}^{\mathrm{XC}}+g_{\mathrm{XC}, \mathrm{ia}, \mathrm{jb}, \mathrm{kl}}^{\mathrm{nadd}}+g_{\mathrm{kin}, \mathrm{ia}, \mathrm{jb}, \mathrm{kl}}^{\mathrm{nadd}}\right] \\
& \times(X+Y)_{\mathrm{ia}}(X+Y)_{\mathrm{jb}},
\end{aligned}
$$

then the first two terms are identical to the left-hand side of eqn (156) (see Section B.1), and $Q_{\mathrm{kl}}$ finally reads

$$
\begin{aligned}
Q_{\mathrm{kl}}= & \omega \sum_{\mathrm{a}}\left\{(X-Y)_{\mathrm{ka}}(X+Y)_{\mathrm{la}}+(X-Y)_{\mathrm{la}}(X+Y)_{\mathrm{ka}}\right\} \\
& -\sum_{\mathrm{a}} \varepsilon_{\mathrm{a}}\left\{(X+Y)_{\mathrm{ka}}(X+Y)_{\mathrm{la}}+(X-Y)_{\mathrm{ka}}(X-Y)_{\mathrm{la}}\right\} \\
& +H_{\mathrm{kl}}+[\mathbf{T}]+2 \sum_{\mathrm{ia}, \mathrm{jb}}\left[g_{\mathrm{ia}, \mathrm{jb}, \mathrm{kl}}^{\mathrm{XC}}+g_{\mathrm{XC}, \mathrm{ia}, \mathrm{jb}, \mathrm{kl}}^{\mathrm{nadd}}+g_{\mathrm{kin}, \mathrm{ia}, \mathrm{jb}, \mathrm{kl} l}^{\mathrm{nadd}}\right] \\
& \times(X+Y)_{\mathrm{ia}}(X+Y)_{\mathrm{jb}} .
\end{aligned}
$$

The second case, in which occupied and virtual orbitals are used, i.e. $\mathrm{p}=\mathrm{k}, \mathrm{q}=\mathrm{c}$, reads,

$$
\begin{aligned}
Q_{\mathrm{kc}}= & \sum_{\mu} \frac{\partial G[\mathbf{X}, \mathbf{Y}, \omega]}{\partial C_{\mu \mathrm{k}}} C_{\mu \mathrm{c}}=2 \sum_{\mathrm{rs}} T_{\mathrm{rs}} F_{\mathrm{cs}} \delta_{\mathrm{rk}} \\
& +\sum_{\mathrm{rs}} T_{\mathrm{rs}} 2\left[(\mathrm{rs} \mid \mathrm{kc})+f_{\mathrm{rs}, \mathrm{kc}}^{\mathrm{XC}}+f_{\mathrm{XC}, \mathrm{rs}, \mathrm{kc}}^{\mathrm{nadd}}+f_{\mathrm{kin}, \mathrm{rs}, \mathrm{kc}}^{\mathrm{nadd}}\right] \\
& +\sum_{\mathrm{ia}, \mathrm{jb}}(X+Y)_{\mathrm{ia}}(X+Y)_{\mathrm{jb}} \\
& \times 2\left[(\mathrm{ca} \mid \mathrm{jb})+f_{\mathrm{ca}, \mathrm{jb}}^{\mathrm{XC}}+f_{\mathrm{XC}, \mathrm{ca}, \mathrm{jb}}^{\mathrm{nadd}}+f_{\mathrm{kin}, \mathrm{ca}, \mathrm{jb}}^{\mathrm{nadd}}\right] \delta_{\mathrm{ik}} \\
& +2 \sum_{\mathrm{ia}, \mathrm{jb}}\left[g_{\mathrm{ia}, \mathrm{jb}, \mathrm{kc}}^{\mathrm{XC}}+g_{\mathrm{XC}, \mathrm{ia}, \mathrm{jb}, \mathrm{kc}}^{\mathrm{nadd}}+g_{\mathrm{kin}, \mathrm{ia}, \mathrm{jb}, \mathrm{kc}}^{\mathrm{nadd}}\right](X+Y)_{\mathrm{ia}}(X+Y)_{\mathrm{jb}} \\
= & 2 \underbrace{T_{\mathrm{kc}}}_{0} \varepsilon_{\mathrm{c}}+H_{\mathrm{kc}}+[\mathbf{T}]+\sum_{\mathrm{a}, \mathrm{jb}}(X+Y)_{\mathrm{ka}}(X+Y)_{\mathrm{jb}} \\
& \times 2\left[(\mathrm{ca} \mid \mathrm{jb})+f_{\mathrm{ca}, \mathrm{jb}}^{\mathrm{XC}}+f_{\mathrm{XC}, \mathrm{ca}, \mathrm{jb}}^{\mathrm{nadd}}+f_{\mathrm{kin}, \mathrm{ca}, \mathrm{jb}}^{\mathrm{nadd}}\right] \\
& +2 \sum_{\mathrm{ia}, \mathrm{jb}}\left[g_{\mathrm{ia}, \mathrm{jb}, \mathrm{kc}}+g_{\mathrm{XC}, \mathrm{ia}, \mathrm{jb}, \mathrm{kc}}^{\mathrm{nadd}}+g_{\mathrm{kin}, \mathrm{i} \mathrm{j}, \mathrm{jb}, \mathrm{kc}}^{\mathrm{nadd}}\right](X+Y)_{\mathrm{ia}}(X+Y)_{\mathrm{jb}}
\end{aligned}
$$

$$
\begin{aligned}
= & \sum_{\mathrm{a}}(X+Y)_{\mathrm{ka}} H_{\mathrm{ca}}{ }^{+}[(\mathbf{X}+\mathbf{Y})]+H_{\mathrm{kc}}{ }^{+}[\mathbf{T}] \\
& +2 \sum_{\mathrm{ia}, \mathrm{jb}}\left[g_{\mathrm{ia}, \mathrm{jb}, \mathrm{kc}}^{\mathrm{XC}}+g_{\mathrm{XC}, \mathrm{ia}, \mathrm{jb}, \mathrm{kc}}^{\mathrm{nadd}}+g_{\mathrm{kin}, \mathrm{ia}, \mathrm{jb}, \mathrm{kc}}^{\mathrm{nadd}}\right] \\
& \times(X+Y)_{\mathrm{ia}}(X+Y)_{\mathrm{jb}} .
\end{aligned}
$$

The transformation made in the last step is valid due to the nature of $\mathbf{X}$ and $\mathbf{Y}\left(X_{\mathrm{aa}}=Y_{\mathrm{aa}}=X_{\mathrm{ii}}=Y_{\mathrm{ii}}=0\right)$ in spite of the fact that the sum is not over pq as in the definition $H_{\mathrm{ca}}{ }^{+}[\mathbf{M}]$, eqn (47), but over jb. The third case is $\mathrm{p}=\mathrm{c}, \mathrm{q}=\mathrm{k}$, 


$$
\begin{aligned}
Q_{\mathrm{ck}}= & \sum_{\mu} \frac{\partial G[\mathbf{X}, \mathbf{Y}, \omega]}{\partial C_{\mu \mathrm{c}}} C_{\mu \mathrm{k}} \\
= & 2 \sum_{\mathrm{rs}} T_{\mathrm{rs}} F_{\mathrm{ks}} \delta_{\mathrm{rc}}+\sum_{\mathrm{ia}, \mathrm{jb}}(X+Y)_{\mathrm{ia}}(X+Y)_{\mathrm{jb}} \\
& \times 2\left[(\mathrm{ik} \mid \mathrm{jb})+f_{\mathrm{ik}, \mathrm{jb}}^{\mathrm{XC}}+f_{\mathrm{XC}, \mathrm{ik}, \mathrm{jb}}^{\mathrm{nadd}}+f_{\mathrm{kin}, \mathrm{ik}, \mathrm{jb}}^{\mathrm{nadd}}\right] \delta_{\mathrm{ac}} \\
= & 2 \underbrace{T_{\mathrm{ck}}}_{0} \varepsilon_{\mathrm{k}}+\sum_{\mathrm{i}, \mathrm{jb}}(X+Y)_{\mathrm{ic}}(X+Y)_{\mathrm{jb}} \\
& \times 2\left[(\mathrm{ik} \mid \mathrm{jb})+f_{\mathrm{ik}, \mathrm{jb}}^{\mathrm{XC}}+f_{\mathrm{XC}, \mathrm{ik}, \mathrm{jb}}^{\text {nadd }}+f_{\text {kin, }, \mathrm{ik}, \mathrm{jb}}^{\mathrm{nadd}}\right] \\
= & \sum_{\mathrm{i}}(X+Y)_{\mathrm{ic}} H_{\mathrm{ik}}+[(\mathbf{X}+\mathbf{Y})] .
\end{aligned}
$$

The last case corresponds to $\mathrm{p}=\mathrm{c} \leq \mathrm{q}=\mathrm{d}$, and results in

$$
\begin{aligned}
Q_{\mathrm{cd}}= & \sum_{\mu} \frac{\partial G[\mathbf{X}, \mathbf{Y}, \omega]}{\partial C_{\mu \mathrm{c}}} C_{\mu \mathrm{d}} \\
= & 2 \sum_{\mathrm{rs}} T_{\mathrm{rs}} F_{\mathrm{ds}} \delta_{\mathrm{rc}}+\sum_{\mathrm{ia}, \mathrm{jb}}(X+Y)_{\mathrm{ia}}(X+Y)_{\mathrm{jb}} \\
& \times 2\left[(\mathrm{id} \mid \mathrm{jb})+f_{\mathrm{id}, \mathrm{jb}}^{\mathrm{XC}}+f_{\mathrm{XC}, \mathrm{id}, \mathrm{jb}}^{\mathrm{nadd}}+f_{\text {kin,id }, \mathrm{jb}}^{\text {nadd }}\right] \delta_{\mathrm{ac}} \\
= & 2 \sum_{\mathrm{s}} T_{\mathrm{cs}} F_{\mathrm{ds}}+\sum_{\mathrm{i}, \mathrm{jb}}(X+Y)_{\mathrm{ic}}(X+Y)_{\mathrm{jb}} \\
& \times 2\left[(\mathrm{id} \mid \mathrm{jb})+f_{\mathrm{id}, \mathrm{jb}}^{\mathrm{XC}}+f_{\mathrm{XC}, \mathrm{id}, \mathrm{jb}}^{\mathrm{nadd}}+f_{\mathrm{kin}, \mathrm{id}, \mathrm{jb}}^{\mathrm{nadd}}\right] \\
= & \varepsilon_{\mathrm{d}} \sum_{\mathrm{i}}\left\{(X+Y)_{\mathrm{ic}}(X+Y)_{\mathrm{id}}+(X-Y)_{\mathrm{ic}}(X-Y)_{\mathrm{id}}\right\} \\
& +\sum_{\mathrm{i}, \mathrm{jb}}(X+Y)_{\mathrm{ic}}(X+Y)_{\mathrm{jb}} \\
& \times 2\left[(\mathrm{id} \mid \mathrm{jb})+f_{\mathrm{id}, \mathrm{jb}}^{\mathrm{XC}}+f_{\mathrm{XC}, \mathrm{id}, \mathrm{jb}}^{\mathrm{nadd}}+f_{\mathrm{kin}, \mathrm{id}, \mathrm{jb}}^{\mathrm{nadd}}\right] .
\end{aligned}
$$

Now one can notice that if $\sum_{\mathrm{i}} \varepsilon_{\mathrm{i}}\left\{(X-Y)_{\mathrm{ic}}(X-Y)_{\text {id }}+(X+Y)_{\mathrm{ic}}(X+Y)_{\mathrm{id}}\right\}$ is added and subtracted from the expression for element $Q_{\mathrm{cd}}$,

$$
\begin{aligned}
Q_{\mathrm{cd}}= & \sum_{\mathrm{i}}\left(\varepsilon_{\mathrm{d}}-\varepsilon_{\mathrm{i}}\right)\left\{(X+Y)_{\mathrm{ic}}(X+Y)_{\mathrm{id}}+(X-Y)_{\mathrm{ic}}(X-Y)_{\mathrm{id}}\right\} \\
& +\sum_{\mathrm{i}, \mathrm{jb}}(X+Y)_{\mathrm{ic}}(X+Y)_{\mathrm{jb}} \\
& \times 2\left[(\mathrm{id} \mid \mathrm{jb})+f_{\mathrm{id}, \mathrm{jb}}^{\mathrm{XC}}+f_{\mathrm{XC}, \mathrm{id}, \mathrm{jb}}^{\mathrm{nadd}}+f_{\mathrm{kin}, \mathrm{id}, \mathrm{jb}}^{\text {nadd }}\right] \\
& +\sum_{\mathrm{i}} \varepsilon_{\mathrm{i}}\left\{(X-Y)_{\mathrm{ic}}(X-Y)_{\mathrm{id}}+(X+Y)_{\mathrm{ic}}(X+Y)_{\mathrm{id}}\right\},
\end{aligned}
$$

then the first two terms are identical to the left-hand side of eqn (157) (see Section B.1), and $Q_{c d}$ finally reads

$$
\begin{aligned}
Q_{\mathrm{cd}}= & \sum_{\mathrm{i}} \omega\left\{(X+Y)_{\mathrm{ic}}(X-Y)_{\mathrm{id}}+(X-Y)_{\mathrm{ic}}(X+Y)_{\mathrm{id}}\right\} \\
& +\sum_{\mathrm{i}} \varepsilon_{\mathrm{i}}\left\{(X-Y)_{\mathrm{ic}}(X-Y)_{\mathrm{id}}+(X+Y)_{\mathrm{ic}}(X+Y)_{\mathrm{id}}\right\} .
\end{aligned}
$$

At this point, all elements of the $\mathbf{Q}$ vector are determined, and the elements of vector $\mathbf{R}$ from the $Z$-vector equation, eqn (144), can be expressed in the following way

$$
\begin{aligned}
R_{\mathrm{kc}}= & -\left(Q_{\mathrm{kc}}-Q_{\mathrm{ck}}\right)=-\left(\sum_{\mathrm{a}}(X+Y)_{\mathrm{ka}} H_{\mathrm{ca}}{ }^{+}[(\mathbf{X}+\mathbf{Y})]\right. \\
& -\sum_{\mathrm{i}}(X+Y)_{\mathrm{ic}} H_{\mathrm{ik}}^{+}[(\mathbf{X}+\mathbf{Y})]+H_{\mathrm{kc}}{ }^{+}[\mathbf{T}] \\
& +2 \sum_{\mathrm{ia}, \mathrm{jb}}\left[g_{\mathrm{ia}, \mathrm{jb}, \mathrm{kc}}^{\mathrm{XC}}+g_{\mathrm{XC}, \mathrm{ia}, \mathrm{jb}, \mathrm{kc}}^{\mathrm{nadd}}+g_{\mathrm{kin}, \mathrm{ia}, \mathrm{jb}, \mathrm{kc}}^{\mathrm{nadd}}\right. \\
& \left.\left.\times(X+Y)_{\mathrm{ia}}(X+Y)_{\mathrm{jb}}\right]\right) .
\end{aligned}
$$

The form of the elements of matrices $\mathbf{Q}$ and $\mathbf{R}$ presented here is most convenient for practical calculations and was first presented in ref. 5 . The $Z$-vector equation and all relevant variables can also be rewritten in terms of densities. ${ }^{10}$ For that purpose, the quantities $H_{\mathrm{kl}}{ }^{+}[\mathbf{M}], Q_{\mathrm{kl}}$, and $Q_{\mathrm{kc}}$ should be expressed as

$$
\begin{aligned}
H_{\mathrm{kl}}{ }^{+}[\mathbf{M}] & =2 \int \phi_{\mathrm{k}} \phi_{1}\left(v_{\mathrm{C}}^{\mathrm{M}}+\left[f^{\mathrm{XC}}+f_{\mathrm{XC}}^{\mathrm{nadd}}+f_{\mathrm{kin}}^{\mathrm{nadd}}\right] \rho^{\mathrm{M}}\right), \\
Q_{\mathrm{kl}}= & \sum_{\mathrm{a}} \omega\left[(X+Y)_{\mathrm{ka}}(X-Y)_{\mathrm{la}}+(X-Y)_{\mathrm{ka}}(X+Y)_{\mathrm{la}}\right] \\
& -\sum_{\mathrm{a}} \varepsilon_{\mathrm{a}}\left\{(X+Y)_{\mathrm{ka}}(X+Y)_{\mathrm{la}}+(X-Y)_{\mathrm{ka}}(X-Y)_{\mathrm{la}}\right\} \\
& +H_{\mathrm{kl}}{ }^{+}[\mathbf{T}]+2 \int \phi_{\mathrm{k}}\left[g^{\mathrm{XC}}+g_{\mathrm{XC}}^{\mathrm{nadd}}+g_{\mathrm{kin}}^{\mathrm{nadd}}\right] \phi_{1} \rho^{(X+Y)} \rho^{(X+Y)},
\end{aligned}
$$

$Q_{\mathrm{kc}}=\sum_{\mathrm{a}}(X+Y)_{\mathrm{ka}} H_{\mathrm{ca}}{ }^{+}[\mathbf{X}+\mathbf{Y}]$

$$
+2 \int \phi_{\mathrm{k}}\left[g^{\mathrm{XC}}+g_{\mathrm{XC}}^{\mathrm{nadd}}+g_{\mathrm{kin}}^{\mathrm{nadd}}\right] \phi_{\mathrm{c}} \rho^{(X+Y)} \rho^{(X+Y)}+H_{\mathrm{kc}}{ }^{+}[\mathbf{T}] .
$$

$Q_{\text {ck }}$ and $Q_{\text {cd }}$ will stay the same, while $R_{\mathrm{ia}}$ can be rewritten as

$$
\begin{aligned}
R_{\mathrm{kc}}= & -\left(\sum_{\mathrm{a}}\left\{(X+Y)_{\mathrm{kc}} H_{\mathrm{ca}}^{+}[\mathbf{X}+\mathbf{Y}]\right\}\right. \\
& -\sum_{i}\left\{(X+Y)_{\mathrm{ic}} H_{\mathrm{ik}}^{+}[\mathbf{X}+\mathbf{Y}]\right\}+H_{\mathrm{kc}}{ }^{+}[\mathbf{T}] \\
& \left.+2 \int \phi_{\mathrm{k}}\left[g^{\mathrm{XC}}+g_{\mathrm{XC}}^{\mathrm{nadd}}+g_{\mathrm{kin}}^{\mathrm{nadd}}\right] \phi_{\mathrm{c}} \rho^{(X+Y)} \rho^{(X+Y)}\right) .
\end{aligned}
$$

\section{Acknowledgements}

This work was supported by a VIDI grant (700.59.422) of the Netherlands Organization for Scientific Research (NWO). Helpful discussions with the late Tom Ziegler are gratefully acknowledged. 


\section{References}

1 Computational Photochemistry, ed. M. Olivucci, Elsevier, Amsterdam, 2005.

2 J. B. Foresman, M. Head-Gordon, J. A. Pople and M. J. Frisch, Toward a Systematic Molecular Orbital Theory for Excited States, J. Phys. Chem., 1992, 96, 135-149.

$3 \mathrm{~J}$. V. Ortiz, One-electron density matrices and energy gradients in the random phase approximation, J. Chem. Phys., 1994, 101, 6743-6749.

4 C. Van Caillie and R. D. Amos, Geometric derivatives of excitation energies using SCF and DFT, Chem. Phys. Lett., 1999, 308, 249-255.

5 F. Furche and R. Ahlrichs, Adiabatic time-dependent density functional methods for excited state properties, J. Chem. Phys., 2002, 117, 7433-7447.

6 T. Helgaker and P. Jørgenson, Configuration-interaction energy derivatives in a fully variational formulation, Theor. Chim. Acta, 1989, 75, 111-127.

7 J. Hutter, Excited-state nuclear forces from the TammDancoff approximation to time-dependent density functional theory within the plane wave basis set framework, J. Chem. Phys., 2003, 118, 3928-3934.

8 N. L. Doltsinis and D. S. Kosov, Plane wave/pseudopotential implementation of excited state gradients in density functional linear response theory: A new route via implicit differentiation, J. Chem. Phys., 2005, 122, 144101.

9 N. L. Doltsinis and M. Sprik, Electronic excitation spectra from time-dependent density functional response theory using plane-wave methods, Chem. Phys. Lett., 2000, 330, 563-569.

10 M. Seth, G. Mazur and T. Ziegler, Time-dependent density functional theory gradients in the Amsterdam density functional package: geometry optimizations of spin-flip excitations, Theor. Chem. Acc., 2011, 129, 331-342.

11 Amsterdam density functional program. Theoretical Chemistry, Vrije Universiteit, Amsterdam. URL: http://www.scm.com.

12 G. te Velde, F. M. Bickelhaupt, E. J. Baerends, S. J. A. van Gisbergen, C. Fonseca Guerra, J. G. Snijders and T. Ziegler, Chemistry with ADF, J. Comput. Chem., 2001, 22, 931-967.

13 J. Tomasi, B. Mennucci and R. Cammi, Quantum Mechanical Continuum Solvation Models, Chem. Rev., 2005, 105, 2999-3094.

14 D. Bakowies and W. Thiel, Hybrid Models for Combined Quantum Mechanical and Molecular Mechanical Approaches, J. Phys. Chem., 1996, 100, 10580-10594.

15 T. A. Wesolowski and A. Warshel, Frozen Density Functional Approach for $a b$ Initio Calculations of Solvated Molecules, J. Phys. Chem., 1993, 97, 8050.

16 G. Scalmani, M. J. Frisch, B. Mennucci, J. Tomasi, R. Cammi and V. Barone, Geometries and properties of excited states in the gas phase and in solution: Theory and application of a time-dependent density functional theory polarizable continuum model, J. Chem. Phys., 2006, 124, 094107.

17 D. Si and H. Li, Analytic energy gradient in combined timedependent density functional theory and polarizable force field calculation, J. Chem. Phys., 2010, 133(14), 144112.
18 N. Minezawa, N. De Silva, F. Zahariev and M. S. Gordon, Implementation of the analytic energy gradient for the combined time-dependent density functional theory/effective fragment potential method: Application to excited-state molecular dynamics simulations, J. Chem. Phys., 2011, 134, 054111.

19 M. Chiba, D. G. Fedorov, T. Nagata and K. Kitaura, Excited state geometry optimizations by time-dependent density functional theory based on the fragment molecular orbital method, Chem. Phys. Lett., 2009, 474, 227-232.

20 M. E. Casida and T. A. Wesolowski, Generalization of the Kohn-Sham Equations with Constrained Electron Density Formalism and Its Time-Dependent Response Theory Formulation, Int. J. Quantum Chem., 2004, 96, 577-588.

21 T. A. Wesolowski, Hydrogen-bonding induced shifts of the excitation energies in nucleic acid bases: an interplay between electrostatic and electron density overlap effects, J. Am. Chem. Soc., 2004, 126, 11444-11445.

22 J. Neugebauer, M. J. Louwerse, E. J. Baerends and T. A. Wesolowski, The merits of the frozen-density embedding scheme to model solvatochromic shifts, J. Chem. Phys., 2005, 122, 094115.

23 J. Neugebauer, C. R. Jacob, T. A. Wesolowski and E. J. Baerends, An Explicit Quantum Chemical Method for Modeling Large Solvation Shells Applied to Aminocoumarin C151, J. Phys. Chem. A, 2005, 109, 7805-7814.

24 X. Zhou, J. W. Kaminski and T. A. Wesołowski, Multi-scale modelling of solvatochromic shifts from frozen-density embedding theory with non-uniform continuum model of the solvent: the coumarin 153 case, Phys. Chem. Chem. Phys., 2011, 13, 10565-10576.

25 M. Humbert-Droz, X. Zhou, S. V. Shedge and T. A. Wesolowski, How to choose the frozen density in Frozen-Density Embedding Theory-based numerical simulations of local excitations, Theor. Chem. Acc., 2013, 132, 1405.

26 J. Neugebauer, Orbital-Free Embedding Calculations of Electronic Spectra, in Recent Progress in Orbital-Free Density Functional Theory, ed. T. A. Wesolowski and Y. A. Wang, World Scientific, Singapore, 2013, pp. 325-356.

27 J. Neugebauer, Couplings between electronic transitions in a subsystem formulation of time-dependent density functional theory, J. Chem. Phys., 2007, 126, 134116.

28 G. Fradelos, J. W. Kaminski, T. A. Wesołowski and S. Leutwyler, Cooperative Effect of Hydrogen-Bonded Chains in the Environment of a $\pi \rightarrow \pi^{*}$ Chromophore, J. Phys. Chem. A, 2009, 113, 9766-9771.

29 C. R. Jacob and J. Neugebauer, Subsystem Density-Functional Theory, WIREs Comput. Mol. Sci., 2014, 4, 325-362.

30 T. A. Wesolowski, S. Shedge and X. Zhou, Frozen-density embedding strategy for multilevel simulations of electronic structure, Chem. Rev., 2015, 115, 5891-5928.

31 M. Dułak, J. W. Kamiński and T. A. Wesołowski, Equilibrium Geometries of Noncovalently Bound Intermolecular Complexes Derived from Subsystem Formulation of Density Functional Theory, J. Chem. Theory Comput., 2007, 3, 735-745. 
32 G. Senatore and K. R. Subbaswamy, Density dependence of the dielectric constant of rare-gas crystals, Phys. Rev. B: Condens. Matter Mater. Phys., 1986, 34, 5754.

33 P. Cortona, Self-consistently determined properties of solids without band-structure calculations, Phys. Rev. B: Condens. Matter Mater. Phys., 1991, 44, 8454.

34 T. A. Wesolowski and J. Weber, Kohn-Sham equations with constrained electron density: an iterative evaluation of the ground-state electron density of interacting molecules, Chem. Phys. Lett., 1996, 248, 71-76.

35 A. Dreuw and M. Head-Gordon, Single-Reference $a b$ Initio Methods for the Calculation of Excited States of Large Molecules, Chem. Rev., 2005, 105, 4009-4037.

36 C. König, N. Schlüter and J. Neugebauer, Direct determination of exciton couplings from subsystem time-dependent density-functional theory within the tamm-dancoff approximation, J. Chem. Phys., 2013, 138, 034104.

37 N. C. Handy and H. F. Schaefer, III, On the evaluation of analytic energy derivatives for correlated wave functions, J. Chem. Phys., 1984, 81, 5031-5033.

38 U. Ekström, L. Visscher, R. Bast, A. J. Thorvaldsen and K. Ruud, Arbitrary-Order Density Functional Response Theory from Automatic Differentiation, J. Chem. Theory Comput., 2010, 6, 1971-1980.

39 A. D. Becke, Density-functional exchange-energy approximation with correct asymptotic behavior, Phys. Rev. A: At., Mol., Opt. Phys., 1988, 38(6), 3098-3100.

40 J. P. Perdew, Density-functional approximation for the correlation energy of the inhomogeneous electron gas, Phys. Rev. B: Condens. Matter Mater. Phys., 1986, 33, 8822-8824.
41 C. R. Jacob, J. Neugebauer and L. Visscher, A flexible implementation of frozen-density embedding for use in multilevel simulations, J. Comput. Chem., 2008, 29, 1011-1018.

42 T. A. Wesolowski, Density functional theory with approximate kinetic energy functionals applied to hydrogen bonds, J. Chem. Phys., 1997, 106, 8516-8526.

43 J. P. Perdew, in Electronic Structure of Solids '91, ed. P. Ziesche and H. Eschrig, Akademie Verlag, Berlin, 1991, p. 11.

44 A. Kovyrshin and J. Neugebauer, Potential-Energy Surfaces of Local Excited States from Subsystem- and Selective Kohn-Sham-TDDFT, Chem. Phys., 2011, 391, 147.

45 J. Neugebauer, Photophysical Properties of Natural LightHarvesting Complexes Studied by Subsystem Density Functional Theory, J. Phys. Chem. B, 2008, 112, 2207-2217.

46 J. Neugebauer, C. Curutchet, A. Muños-Losa and B. Mennucci, A Subsystem TDDFT Approach for Solvent Screening Effects on Excitation Energy Transfer Couplings, J. Chem. Theory Comput., 2010, 6, 1843-1851.

47 F. Furche and D. Rappoport, Density Functional Methods for Excited States: Equilibrium Structure and Electronic Spectra, in Computational Photochemistry, Volume 16 of Theoretical and Computational Chemistry, ed. M. Olivucci, Elsevier, Amsterdam, 2005, pp. 93-128.

48 D. Rappoport and F. Furche, Analytical time-dependent density functional derivative methods within the RI-J approximation, an approach to excited states of large molecules, J. Chem. Phys., 2005, 122, 064105.

49 Y. Yamaguchi, Y. Osamura, J. D. Goddard and H. F. Schaefer III, A New Dimension to Quantum Chemistry: Analytical Derivative Methods in Ab Initio Molecular Electronic Structure Theory, Oxford University Press, Oxford, 1994. 